Tohoku Math. J.

54 (2002), 419-441

\title{
FLOQUET MULTIPLIERS OF SYMMETRIC RAPIDLY OSCILLATING SOLUTIONS OF DIFFERENTIAL DELAY EQUATIONS
}

\author{
Peter Dormayer, Anatoli F. IVAnOV* And Bernhard Lani-Wayda
}

(Received June 27, 2000, revised February 20, 2002)

\begin{abstract}
Floquet multipliers of symmetric rapidly oscillating periodic solutions of the differential delay equation $\dot{x}(t)=\alpha f(x(t), x(t-1))$ with the symmetries $f(-x, y)=$ $f(x, y)=-f(x,-y)$ are described in terms of zeroes of a characteristic function. A relation to the characteristic function of symmetric slowly oscillating periodic solutions is found. Sufficient conditions for the existence of at least one real multiplier outside the unit disc are derived. An example with a piecewise linear function $f$ is studied in detail, both analytically and numerically.
\end{abstract}

1. Introduction. The approach of exploiting symmetry properties of delay equations and of periodic solutions to study the Floquet multipliers of such solutions is well-known, but was so far mainly used in connection with slowly oscillating solutions. In the present work we extend this method to rapidly oscillating solutions with periods which are commensurable with the delay. We investigate the relations between stability properties of slowly and rapidly oscillating solutions. Although both types of solutions are connected to each other by transformations, the relation between the characteristic functions that we derive in Section 3 is rather nontrivial.

We apply the result to the study of stability, secondary bifurcation, and asymptotic behavior of Floquet multipliers (FM) for rapidly oscillating periodic solutions in Section 4. (Recall that the FM of a periodic solution are the eigenvalues of the derivative of the period map at the initial value of this solution, and thus determine the stability of the solution.) In Section 5, we describe Floquet multipliers for rapidly oscillating periodic solutions of a piecewise linear equation with sine-like feedback. In particular, we obtain a real multiplier oscillating about 1 as a parameter is varied. One might conjecture that this is the dominating multiplier, in which case one would have a simple example for the occurrence of rapidly oscillating, but stable periodic solutions. The fact that rapidly oscillating solutions can be stable at all is a rather recent discovery, see $[10,20]$. However, by numerical investigation we have found that (for the numerically accessible parameter range) there exist non-real multipliers outside the unit circle, so that we do not obtain stability in our example.

2000 Mathematics Subject Classification. Primary 34K13; Secondary 34K18

Key words and phrases. Delay equations with symmetry, rapidly oscillating periodic solutions, stability, Floquet multipliers

*This work was carried out while the author was at University of Ballarat, Ballarat, Victoria 5535, Australia. 
Let us give a brief summary of known results about the existence and stability of symmetric periodic solutions that we will need in the sequel. For proofs and further details see $[1-8,11,13,15,18,21,22]$ and references therein.

Differential delay equations of the type

$$
\dot{x}(t)=f(x(t), x(t-1))
$$

generate a semiflow on the space $C=C^{0}([-1,0], \boldsymbol{R})$, if $f$ is locally Lipschitz continuous. Equation $(f)$ is called symmetric if the function $f$ satisfies the condition

$$
f(-x, y)=f(x, y)=-f(x,-y) \quad \text { for any }(x, y) \in \boldsymbol{R}^{2} .
$$

Under the additional hypothesis of negative feedback

$$
y \cdot f(x, y)<0 \text { for } y \neq 0,
$$

equation $(f)$ is known to possess the so-called special symmetric periodic solutions $[2,6,13]$ (SSPSs for brief). A periodic solution $x: \boldsymbol{R} \rightarrow \boldsymbol{R}$ is called special symmetric if $x(t+2)=$ $-x(t)$ for all $t \in \boldsymbol{R}$. Such a solution has period 4 and is slowly oscillating, that is, the distance between its consecutive zeros is larger than the delay 1 . A solution is called eventually slowly oscillating if there exists time $T$ such that the distance between its consecutive zeros to the right from $T$ is larger than 1. A solution that is not eventually slowly oscillating is called rapidly oscillating.

The existence of SSPSs is well-known [2, 6, 13]. Equation $(f)$ has a SSPS if and only if the system of ordinary differential equations in the plane

$$
\dot{x}=f(x, y) \quad \dot{y}=-f(y, x)
$$

has a symmetric closed trajectory with the minimal period 4 encompassing the origin $(0,0)$. The first component $x(t)$ of this trajectory solves equation $(f)$ and is called the Kaplan-Yorke solution (first introduced and studied in [15]).

A SSPS can be normalized in such a way that $x(0)=0$ and $\dot{x}(0)>0$. The value of $x(1):=z$ is called the amplitude of the SSPS.

The parameterized family of delay differential equations of the form

$$
\dot{x}(t)=\alpha f(x(t), x(t-1)), \quad \alpha \in \boldsymbol{R}
$$

possesses, under some general assumptions, the so-called primary branch $(\mathrm{PB})$ of SSPSs. The PB has the same degree of smoothness as the nonlinearity $f(x, y)$. It bifurcates from zero at $\alpha=-\pi /\left(2 f_{y}(0,0)\right)$ and exists for $z>0$ in at least some vicinity of $z=0[2,6]$.

The stability of SSPSs can be determined from the location of their Floquet multipliers in the complex plane. Floquet multipliers are the eigenvalues of the monodromy operator, the shift by time 4 along solutions, of the corresponding variational equation

$$
\dot{v}(t)=f_{x}(x(t), x(t-1)) v(t)+f_{y}(x(t), x(t-1)) v(t-1),
$$

which we abbreviate as $\dot{v}(t)=a(t) v(t)+b(t) v(t-1)$. Due to the symmetries $(s)$ the coefficients $a$ and $b$ of equation (ve) are periodic with period 2. The eigenvalues of the shift operator by time 2 along the solutions of equation (ve) are called semi-Floquet multipliers. 
The Floquet multipliers are given then as the squares of semi-Floquet multipliers. The nonzero semi-Floquet multipliers can be completely described in terms of zeroes of an analytic function, called the characteristic function $[2,6,21,22]$. The characteristic function has been employed to study the stability of SSPSs for several classes of symmetric delay differential equations $[1,2,6,7,11,22]$.

The present paper deals with the symmetric rapidly oscillating periodic solutions (abbreviated as SROSs) of equation $(f)$. A periodic solution $x(t)$ is called symmetric if $x(t+\omega)=$ $-x(t)$ for all $t \in \boldsymbol{R}$ and some $\omega>0$. In the case $\omega=2$ one obtains the above described SSPSs. If $\omega<1$, the corresponding periodic solution of period $2 \omega$ is rapidly oscillating.

Some SROSs can be obtained as transformed SSPSs via the so-called Cooke [14] or Saupe [19] transformations. (Details of these transformations are given in Section 2.) The periods of the SROSs are given by $2 \omega=4 /(2 n+1), n \in N$, and therefore are commensurable with the delay 1 . This allows one to apply the same idea as in the case of the SSPSs to characterize their Floquet multipliers in terms of an analytic function. The characteristic function for the SROSs is derived in Section 3. A relation to the characteristic function of the SSPSs is found (Theorem 3.3).

A further description of the Floquet multipliers of the SROSs is obtained in Section 4, where conditions for the existence of a real Floquet multiplier outside the unit disk are given. Secondary bifurcations of SROSs, which are related to SSPSs on the primary branch by transformation, are discussed. The asymptotic behavior as $\alpha \rightarrow \infty$ of the semi-Floquet multipliers of the SROSs is studied for a class of equations of the form $(f)$ with only a single delay term.

In Section 5, we study an example with a piecewise linear nonlinearity $f$, where we can obtain more explicit information on the semi-Floquet multipliers of SROSs.

This research was initiated during the second author's research visit to the Justus-LiebigUniversität, Giessen, under the support of the Alexander-von-Humboldt-Stiftung, Germany (1998). It was continued during a research stay of all three authors at the Mathematisches Forschungsinstitut Oberwolfach within its program "Research in Pairs" supported by the Volkswagen-Stiftung (1999), and completed 2000. The second author was also partially supported by the Australian Research Council.

2. Rapidly oscillating periodic solutions and transformations. We consider the differential delay equation

$$
\dot{x}(t)=f(x(t), x(t-1)),
$$

where we assume that $f$

- is continuously differentiable,

- is even with respect to the first argument and odd with respect to the second argument,

- satisfies the feedback condition $y \cdot f(x, y) \neq 0$ (at least for $(x, y)$ in a neighborhood of $(0,0)$ in $\left.\boldsymbol{R}^{2}\right)$.

Let $x: \boldsymbol{R} \rightarrow \boldsymbol{R}$ be a special symmetric solution of equation $(f)$, i.e., 
$-x$ is a solution of equation $(f)$,

$-x$ has the symmetry property $x(t+2)=-x(t)(t \in \boldsymbol{R})$,

$-x$ is odd.

Observe that then $x(t+2 n)=(-1)^{n} x(t)$ for $n \in \boldsymbol{Z}, t \in \boldsymbol{R}$. Conditions for the existence of such solutions can be found in [7, 12]. By Cooke's and Saupe's groups of transformations $[14,19]$ we find rapidly oscillating periodic solutions of transformed equations: For $n \in Z$, set $\alpha_{n}:=2 n+1, x_{n}(t):=x\left(\alpha_{n} t\right)(t \in \boldsymbol{R})$. Then

$$
x\left(\alpha_{n} t-1\right)=(-1)^{n} x\left(\alpha_{n} t-1-2 n\right)=(-1)^{n} x\left(\alpha_{n} t-\alpha_{n}\right)=(-1)^{n} x_{n}(t-1),
$$

and

$$
\begin{aligned}
\dot{x}_{n}(t) & =\alpha_{n} \dot{x}\left(\alpha_{n} t\right)=\alpha_{n} f\left(x\left(\alpha_{n} t\right), x\left(\alpha_{n} t-1\right)\right)=\alpha_{n} f\left(x_{n}(t),(-1)^{n} x_{n}(t-1)\right) \\
& =(-1)^{n} \alpha_{n} f\left(x_{n}(t), x_{n}(t-1)\right) .
\end{aligned}
$$

Thus we obtain that

$-x_{n}$ solves equation $\left(f_{n}\right)$, where $f_{n}:=(-1)^{n} \alpha_{n} f$,

$-x_{n}$ has the symmetry property $x_{n}\left(t+2 / \alpha_{n}\right)=-x_{n}(t)$,

$-x_{n}$ is periodic with period $4 / \alpha_{n}$.

Observe that $f_{n}$ and $f$ have the opposite type of feedback for odd $n>0$, and also for even $n<0$. Note also that $x_{-n}=-x_{n-1}$, since $x$ is odd.

It is clear that if $y: \boldsymbol{R} \rightarrow \boldsymbol{R}$ is a solution of equation $(f)$ with the symmetry $y(t+$ $\left.2 / \alpha_{n}\right)=-y(t)(t \in \boldsymbol{R})$, then $x(t):=y\left(t / \alpha_{n}\right)$ defines a special symmetric solution of equation $\left((-1)^{n}\left(1 / \alpha_{n}\right) f\right)$.

REMARK. If $x$ is a solution of equation $(f)$ with the symmetry $x(t+\omega / 2)=-x(t)$ $(t \in \boldsymbol{R})$, and if $n \in \boldsymbol{Z}$ is such that $n \omega+2 \neq 0$, then $x_{n}(t):=x((1+n \omega / 2) t)$ defines a solution of equation $\left(f_{n}\right)$, where now $f_{n}:=(-1)^{n}(1+n \omega / 2) f$; this solution is also symmetric with period $\omega_{n}:=2 \omega /(n \omega+2)$. This is Saupe's transformation $\mathcal{S}_{n}$. For even $n$, we have Cooke's transformation $\mathcal{C}_{n}$; these transformations apply to any periodic solution, without symmetry conditions on $f$ or the solution.

In this paper we study Floquet multipliers of rapidly oscillating solutions of delay equations of type $(f)$, which are derived from special symmetric solutions via Saupe's transformations.

3. Characteristic functions. Let $x$ be a special symmetric solution of $(f)$ and, as above, set $\alpha_{n}:=2 n+1, x_{n}:=x\left(\alpha_{n} \cdot\right)$, and $f_{n}:=(-1)^{n} \alpha_{n} f$ for $n \in N_{0}$. The variational equation along $x_{n}$ is given by $\dot{v}(t)=f_{n, x}\left(x_{n}(t), x_{n}(t-1)\right) v(t)+f_{n, y}\left(x_{n}(t), x_{n}(t-1)\right) v(t-$ 1) $(t \geq 1)$, where the subscripts $x$ and $y$ denote partial differentiation. Define $y_{n}:=x_{n}(\cdot-1)$ and $y:=y_{0}=x(\cdot-1)$. Then

$$
y_{n}(t)=x\left(\alpha_{n} t-\alpha_{n}\right)=x\left(\alpha_{n} t-2 n-1\right)=(-1)^{n} x\left(\alpha_{n} t-1\right)=(-1)^{n} y\left(\alpha_{n} t\right) \quad(t \in \boldsymbol{R}) .
$$

Then, with $a_{n}:=f_{x}\left(x_{n}(\cdot), x_{n}(\cdot-1)\right)=f_{x}\left(x_{n}(\cdot), y_{n}(\cdot)\right)$, we have

$$
f_{n, x}\left(x_{n}(t), x_{n}(t-1)\right)=(-1)^{n} \alpha_{n} f_{x}\left(x_{n}(t), y_{n}(t)\right)=(-1)^{n} \alpha_{n} a_{n}(t) .
$$


Similarly, with $b_{n}:=f_{y}\left(x_{n}(\cdot), x_{n}(\cdot-1)\right)=f_{y}\left(x_{n}(\cdot), y_{n}(\cdot)\right)$, one has

$$
f_{n, y}\left(x_{n}(t), x_{n}(t-1)\right)=(-1)^{n} \alpha_{n} b_{n}(t) .
$$

Hence the variational equation along $x_{n}$ can be rewritten as

$\left(v_{n}\right)$

$$
\dot{v}(t)=(-1)^{n} \alpha_{n}\left[a_{n}(t) v(t)+b_{n}(t) v(t-1)\right] \quad(t \geq 1) .
$$

Let us state some properties of $a_{n}$ and $b_{n}$, and set $\tau_{n}:=1 / \alpha_{n}=1 /(2 n+1)$, and $c_{n}:=$ $b_{n}\left(\cdot+\tau_{n}\right), \quad d_{n}:=a_{n}\left(\cdot+\tau_{n}\right)$. Observe that $f_{x}$ is odd and $f_{y}$ is even in both arguments. Because of

$$
\begin{aligned}
& x_{n}\left(t+\tau_{n}\right)=x\left(\alpha_{n} t+\alpha_{n} \tau_{n}\right)=x\left(\alpha_{n} t+1\right)=-x\left(\alpha_{n} t-1\right)=-(-1)^{n} y_{n}(t), \\
& y_{n}\left(t+\tau_{n}\right)=(-1)^{n} y\left(\alpha_{n}\left(t+\tau_{n}\right)\right)=(-1)^{n} y\left(\alpha_{n} t+1\right)=(-1)^{n} x\left(\alpha_{n} t\right)=(-1)^{n} x_{n}(t),
\end{aligned}
$$

we have

$$
\begin{aligned}
d_{n}(t) & =a_{n}\left(t+\tau_{n}\right)=f_{x}\left(x_{n}\left(t+\tau_{n}\right), y_{n}\left(t+\tau_{n}\right)\right)=f_{x}\left(-(-1)^{n} y_{n}(t),(-1)^{n} x_{n}(t)\right) \\
& =-f_{x}\left(y_{n}(t), x_{n}(t)\right)
\end{aligned}
$$

and, analogously, $c_{n}(t)=f_{y}\left(y_{n}(t), x_{n}(t)\right)$. Since $x_{n}\left(t+2 \tau_{n}\right)=-x_{n}(t)$ and $y_{n}\left(t+2 \tau_{n}\right)=$ $-y_{n}(t)$, we find

$$
\begin{aligned}
a_{n}\left(t+2 \tau_{n}\right) & =f_{x}\left(x_{n}\left(t+2 \tau_{n}\right), y_{n}\left(t+2 \tau_{n}\right)\right)=f_{x}\left(-x_{n}(t),-y_{n}(t)\right) \\
& =f_{x}\left(x_{n}(t), y_{n}(t)\right)=a_{n}(t),
\end{aligned}
$$

i.e., $a_{n}$ is $2 \tau_{n}$-periodic. In the same way we see that this holds for the functions $b_{n}, c_{n}$ and $d_{n}$. As a consequence, we have

$$
\begin{gathered}
a_{n}(t+1)=a_{n}\left(t+(2 n+1) \tau_{n}\right)=a_{n}\left(t+2 n \tau_{n}+\tau_{n}\right)=a_{n}\left(t+\tau_{n}\right)=d_{n}(t), \\
a_{n}\left(t+1+\tau_{n}\right)=a_{n}\left(t+2(n+1) \tau_{n}\right)=a_{n}(t)
\end{gathered}
$$

and, analogously, $b_{n}(t+1)=c_{n}(t), b_{n}\left(t+1+\tau_{n}\right)=b_{n}(t)$. Set $C:=C^{0}([-1,0], \boldsymbol{R})$. For $t, \tau \in \boldsymbol{R}, t \geq \tau$, define the evolution operator $U(t, \tau) \in L_{c}(C, C)$ by $U(t, \tau) \psi=$ $v_{t}^{\psi, \tau}$, where $v^{\psi, \tau}$ is the solution of equation $\left(v_{n}\right)$ with initial condition $v_{\tau}^{\psi, \tau}=\psi$, and the subscripts $t, \tau$ denote segments, as usual. These operators have the property $U(t, s) U(s, \tau)=$ $U(t, \tau)$ for $t \geq s \geq \tau$. Let $V$ be the monodromy operator of $x_{n}$. Then $V=U\left(4 \tau_{n}, 0\right)=$ $U\left(4 \tau_{n}, 2 \tau_{n}\right) U\left(2 \tau_{n}, 0\right)$. The symmetries imply that $U\left(4 \tau_{n}, 2 \tau_{n}\right)=U\left(2 \tau_{n}, 0\right)$, so we have $V=\left[U\left(2 \tau_{n}, 0\right)\right]^{2}$. Periodicity of $x_{n}$ implies that $V^{2 n+1}=\left[U\left(4 \tau_{n}, 0\right)\right]^{2 n+1}=U((2 n+1)$. $\left.4 \tau_{n}, 2 n \cdot 4 \tau_{n}\right) U\left(2 n \cdot 4 \tau_{n},(2 n-1) \cdot 4 \tau_{n}\right) \cdots U\left(4 \tau_{n}, 0\right)=U\left((2 n+1) \cdot 4 \tau_{n}, 0\right)=U(4,0)$. Since the latter operator is compact, all nonzero Floquet multipliers of $x_{n}$ are eigenvalues of $V$. Further, since $V=\left[U\left(2 \tau_{n}, 0\right)\right]^{2}$, the eigenvalues of $U\left(2 \tau_{n}, 0\right)$ are semi-Floquet multipliers of $x_{n}$ (their squares are Floquet multipliers). We want to derive a characteristic function for these semi-Floquet multipliers. To this end, let $\lambda \in C \backslash\{0\}$ be a semi-Floquet multiplier of $x_{n}$. Then there is a solution $v$ of equation $\left(v_{n}\right)$, defined on $\boldsymbol{R}_{0}^{+}$, such that $v\left(t+2 \tau_{n}\right)=\lambda v(t)(t \geq 0)$, which implies

$$
v\left(t+2 k \tau_{n}\right)=\lambda^{k} v(t) \quad\left(t \geq 0, k \in N_{0}\right)
$$


Let $u(t):=v(t+1), w(t):=v\left(t+1+\tau_{n}\right)(t \geq 0)$. Then $u$ and $v$ are differentiable, and the identity $\lambda^{n+1} v(t)=v\left(t+(2 n+2) \tau_{n}\right)=v\left(t+1+\tau_{n}\right)=w(t)$ yields

$$
\begin{aligned}
\dot{u}(t)=\dot{v}(t+1) & =(-1)^{n} \alpha_{n}\left[a_{n}(t+1) v(t+1)+b_{n}(t+1) v(t)\right] \\
& =(-1)^{n} \alpha_{n}\left[d_{n}(t) u(t)+c_{n}(t) \frac{1}{\lambda^{n+1}} w(t)\right] .
\end{aligned}
$$

Analogously, the identity $\lambda^{n} v\left(t+\tau_{n}\right)=v\left(t+\tau_{n}+2 n \tau_{n}\right)=v(t+1)=u(t)$ gives

$$
\begin{aligned}
\dot{w}(t) & =\dot{v}\left(t+1+\tau_{n}\right) \\
& =(-1)^{n} \alpha_{n}\left[a_{n}\left(t+1+\tau_{n}\right) v\left(t+1+\tau_{n}\right)+b_{n}\left(t+1+\tau_{n}\right) v\left(t+\tau_{n}\right)\right] \\
& =(-1)^{n} \alpha_{n}\left[a_{n}(t) w(t)+b_{n}(t) \frac{1}{\lambda^{n}} u(t)\right],
\end{aligned}
$$

or in matrix form

$$
\left(\begin{array}{l}
\dot{w}(t) \\
\dot{u}(t)
\end{array}\right)=(-1)^{n} \alpha_{n} A_{n}(t, \lambda)\left(\begin{array}{l}
w(t) \\
u(t)
\end{array}\right), \quad A_{n}(t, \lambda):=\left(\begin{array}{cc}
a_{n}(t) & \lambda^{-n} b_{n}(t) \\
\lambda^{-(n+1)} c_{n}(t) & d_{n}(t)
\end{array}\right) .
$$

Since $w\left(\tau_{n}\right)=v\left(1+2 \tau_{n}\right)=\lambda v(1)=\lambda u(0)$ and $u\left(\tau_{n}\right)=v\left(1+\tau_{n}\right)=w(0)$, we have the boundary conditions

$$
\left(\begin{array}{l}
w\left(\tau_{n}\right) \\
u\left(\tau_{n}\right)
\end{array}\right)=\left(\begin{array}{c}
\lambda u(0) \\
w(0)
\end{array}\right)=C(\lambda)\left(\begin{array}{l}
w(0) \\
u(0)
\end{array}\right), \quad \text { with } C(\lambda):=\left(\begin{array}{ll}
0 & \lambda \\
1 & 0
\end{array}\right) .
$$

Let $S_{n}(\cdot, \lambda)$ be the fundamental solution of the linear equation above, i.e.,

$$
\dot{S}_{n}(t, \lambda)=(-1)^{n} \alpha_{n} A_{n}(t, \lambda) S_{n}(t, \lambda), \quad S_{n}(0, \lambda)=\left(\begin{array}{ll}
1 & 0 \\
0 & 1
\end{array}\right) .
$$

In view of the periodicity of the coefficient functions, we can extend the set of $t$-values for which $A_{n}(t, \lambda)$ and $S_{n}(t, \lambda)$ are defined to all of $\boldsymbol{R}$. Now one has

$$
\left(\begin{array}{l}
w\left(\tau_{n}\right) \\
u\left(\tau_{n}\right)
\end{array}\right)=S_{n}\left(\tau_{n}, \lambda\right)\left(\begin{array}{l}
w(0) \\
u(0)
\end{array}\right), \quad \text { i.e., } \quad\left[S_{n}\left(\tau_{n}, \lambda\right)-C(\lambda)\right]\left(\begin{array}{l}
w(0) \\
u(0)
\end{array}\right)=0 .
$$

Conversely, assume that ${ }^{t}(\xi, \eta)$ is a nontrivial solution of the equation $\left[S_{n}\left(\tau_{n}, \lambda\right)-\right.$ $C(\lambda)]^{t}(\xi, \eta)=0$. Then we define

$$
\left(\begin{array}{l}
w(t) \\
u(t)
\end{array}\right):=S_{n}(t, \lambda)\left(\begin{array}{l}
\xi \\
\eta
\end{array}\right) \text { for } t \in \boldsymbol{R},
$$

and thus $w\left(\tau_{n}\right)=\lambda u(0), u\left(\tau_{n}\right)=w(0)$. Using the definitions of the functions $c_{n}$ and $d_{n}$, and the $2 \tau_{n}$-periodicity of $a_{n}$ and $b_{n}$, one sees that the functions ${ }^{t}\left(w\left(\cdot+\tau_{n}\right), u\left(\cdot+\tau_{n}\right)\right)$ and ${ }^{t}(\lambda u(\cdot), w(\cdot))$ satisfy the same initial value problem. It follows that $w(t)=u\left(t+\tau_{n}\right)$ and $w\left(t+\tau_{n}\right)=\lambda u(t)$ for $t \in \boldsymbol{R}$, so $u\left(t+2 \tau_{n}\right)=\lambda u(t)$. Set $v(t):=u(t-1)$ for $t \in \boldsymbol{R}$. Then we have $v\left(\cdot+2 \tau_{n}\right)=\lambda v(\cdot)$. Furthermore, $v$ is a nonzero solution of the variational equation $\left(v_{n}\right)$, since

$$
u\left(t+\tau_{n}-1\right)=u\left(t-2+(2 n+2) \tau_{n}\right)=\lambda^{n+1} u(t-2)=\lambda^{n+1} v(t-1),
$$


and hence

$$
\begin{aligned}
\dot{v}(t) & =\dot{u}(t-1)=(-1)^{n} \alpha_{n}\left[\lambda^{-(n+1)} c_{n}(t-1) w(t-1)+d_{n}(t-1) u(t-1)\right] \\
& =(-1)^{n} \alpha_{n}\left[\lambda^{-(n+1)} b_{n}(t) u\left(t+\tau_{n}-1\right)+a_{n}(t) u(t-1)\right] \\
& =(-1)^{n} \alpha_{n}\left[b_{n}(t) v(t-1)+a_{n}(t) v(t)\right] .
\end{aligned}
$$

It then follows that $\lambda$ is a semi-multiplier of $x_{n}$.

Summing up, we have shown the following result.

3.1. LEMMA. $\lambda \in \boldsymbol{C} \backslash\{0\}$ is a semi-Floquet multiplier of $x_{n}$ if and only if

$$
r_{n}(\lambda):=\operatorname{det}\left[S_{n}\left(\tau_{n}, \lambda\right)-C(\lambda)\right]=0 .
$$

Hence $r_{n}$ is a characteristic function for semi-multipliers of $x_{n}$. In the next step we want to relate $r_{n}$ to the characteristic function $r=r_{0}$ of the slowly oscillating solution $x\left(=x_{0}\right)$ of equation $(f)$. To this end, observe that $x$ is odd and $y$ is even, and thus

$$
\begin{aligned}
& x_{n}\left((-1)^{n} t / \alpha_{n}\right)=x\left((-1)^{n} t\right)=(-1)^{n} x(t), \\
& y_{n}\left((-1)^{n} t / \alpha_{n}\right)=(-1)^{n} y\left((-1)^{n} t\right)=(-1)^{n} y(t),
\end{aligned}
$$

and thus we have (with $a:=a_{0}$ etc.)

$$
\begin{aligned}
& a_{n}\left((-1)^{n} t / \alpha_{n}\right)=f_{x}\left((-1)^{n} x(t),(-1)^{n} y(t)\right)=f_{x}(x(t), y(t))=a(t), \\
& b_{n}\left((-1)^{n} t / \alpha_{n}\right)=f_{y}\left((-1)^{n} x(t),(-1)^{n} y(t)\right)=f_{y}(x(t), y(t))=b(t), \\
& c_{n}\left((-1)^{n} t / \alpha_{n}\right)=f_{y}\left((-1)^{n} y(t),(-1)^{n} x(t)\right)=f_{y}(x(t), y(t))=c(t), \\
& d_{n}\left((-1)^{n} t / \alpha_{n}\right)=-f_{x}\left((-1)^{n} y(t),(-1)^{n} x(t)\right)=-f_{x}(y(t), x(t))=d(t) .
\end{aligned}
$$

It follows that $A_{n}\left((-1)^{n} t / \alpha_{n}, \lambda\right)=\left(\begin{array}{cc}a(t) & \lambda^{-n} b(t) \\ \lambda^{-(n+1)} c(t) & d(t)\end{array}\right)$.

$$
\begin{aligned}
& \text { Let } A:=A_{0} \text {, and } D_{n}(\lambda):=\left(\begin{array}{cc}
1 & 0 \\
0 & \lambda^{n}
\end{array}\right), \quad \bar{S}_{n}(t, \lambda):=D_{n}(\lambda)^{-1} S_{n}\left((-1)^{n} t / \alpha_{n}, \lambda\right) \text {. Then } \\
& \qquad \begin{aligned}
\dot{\bar{S}}_{n}(t, \lambda) & =D_{n}(\lambda)^{-1} \frac{(-1)^{n}}{\alpha_{n}} \dot{S}_{n}\left((-1)^{n} t / \alpha_{n}, \lambda\right) \\
& =D_{n}(\lambda)^{-1} A_{n}\left((-1)^{n} t / \alpha_{n}, \lambda\right) S_{n}\left((-1)^{n} t / \alpha_{n}, \lambda\right) \\
& =\bar{A}_{n}(t, \lambda) \bar{S}_{n}(t, \lambda),
\end{aligned}
\end{aligned}
$$

with

$$
\begin{aligned}
\bar{A}_{n}(t, \lambda) & :=D_{n}(\lambda)^{-1} A_{n}\left((-1)^{n} t / \alpha_{n}, \lambda\right) D_{n}(\lambda) \\
& =\left(\begin{array}{cc}
1 & 0 \\
0 & 1 / \lambda^{n}
\end{array}\right)\left(\begin{array}{cc}
a(t) & \lambda^{-n} b(t) \\
\lambda^{-(n+1)} c(t) & d(t)
\end{array}\right)\left(\begin{array}{cc}
1 & 0 \\
0 & \lambda^{n}
\end{array}\right) \\
& =\left(\begin{array}{cc}
1 & 0 \\
0 & 1 / \lambda^{n}
\end{array}\right)\left(\begin{array}{cc}
a(t) & b(t) \\
\lambda^{-(n+1)} c(t) & \lambda^{n} d(t)
\end{array}\right)=\left(\begin{array}{cc}
a(t) & b(t) \\
\lambda^{-(2 n+1)} c(t) & d(t)
\end{array}\right) \\
& =A\left(t, \lambda^{2 n+1}\right) .
\end{aligned}
$$


(We have used that $A(t, \mu)=A_{0}(t, \mu)=\left(\begin{array}{cc}a_{0}(t) & b_{0}(t) \\ \mu^{-1} c_{0}(t) & d_{0}(t)\end{array}\right)$.) Hence we can conclude $\bar{S}_{n}(t, \lambda)=S\left(t, \lambda^{2 n+1}\right) \bar{S}_{n}(0, \lambda)$ or

$$
S_{n}\left((-1)^{n} t / \alpha_{n}, \lambda\right)=D_{n}(\lambda) \bar{S}_{n}(t, \lambda)=D_{n}(\lambda) S\left(t, \lambda^{2 n+1}\right) D_{n}(\lambda)^{-1} .
$$

For $t=(-1)^{n}$ we arrive at the following result.

3.2. Lemma. For $\lambda \in \boldsymbol{C} \backslash\{0\}$ and $n \in \boldsymbol{N}_{0}$ one has

$$
S_{n}\left(\tau_{n}, \lambda\right)=D_{n}(\lambda) S\left((-1)^{n}, \lambda^{2 n+1}\right) D_{n}(\lambda)^{-1} .
$$

We can thus describe $S_{n}\left(\tau_{n}, \lambda\right)$ by $S\left(1, \lambda^{2 n+1}\right)$ or by $S\left(-1, \lambda^{2 n+1}\right)$, depending on whether $n$ is even or odd. We want to express $S_{n}\left(\tau_{n}, \lambda\right)$ by $S\left(1, \lambda^{2 n+1}\right)$ in both cases, so we need a relation between $S(1, \mu)$ and $S(-1, \mu)$ for $\mu \in \boldsymbol{C} \backslash\{0\}$. For fixed $\mu$, set $\tilde{S}(t):=C(\mu) S(t+$ $1, \mu)(t \in \boldsymbol{R})$. Then, using the definitions of $c=c_{0}, d=d_{0}$, and $\tau_{0}=1$ and 2-periodicity of $a, b, c, d$, we get

$$
\dot{\tilde{S}}(t)=C(\mu) \dot{S}(t+1, \mu)=C(\mu) A(t+1, \mu) S(t+1, \mu)=\tilde{A}(t, \mu) \tilde{S}(t),
$$

where

$$
\begin{aligned}
\tilde{A}(t, \mu) & =C(\mu) A(t+1, \mu) C(\mu)^{-1}=C(\mu)\left(\begin{array}{cc}
a(t+1) & b(t+1) \\
c(t+1) / \mu & d(t+1)
\end{array}\right) C(\mu)^{-1} \\
& =\left(\begin{array}{ll}
0 & \mu \\
1 & 0
\end{array}\right)\left(\begin{array}{cc}
d(t) & c(t) \\
b(t) / \mu & a(t)
\end{array}\right)\left(\begin{array}{cc}
0 & 1 \\
1 / \mu & 0
\end{array}\right) \\
& =\left(\begin{array}{cc}
b(t) & \mu a(t) \\
d(t) & c(t)
\end{array}\right)\left(\begin{array}{cc}
0 & 1 \\
1 / \mu & 0
\end{array}\right)=\left(\begin{array}{cc}
a(t) & b(t) \\
c(t) / \mu & d(t)
\end{array}\right) \\
& =A(t, \mu) .
\end{aligned}
$$

Thus $\tilde{S}(t)=S(t, \mu) \tilde{S}(0)$, or $C(\mu) S(t+1, \mu)=S(t, \mu) C(\mu) S(1, \mu)$ for $t \in \boldsymbol{R}$. Now $t=-1$ gives

$$
S(-1, \mu)=C(\mu) S(1, \mu)^{-1} C(\mu)^{-1} .
$$

Combining this relation with $D_{n}(\lambda) C\left(\lambda^{2 n+1}\right)=\lambda^{n} C\left(\lambda^{n+1}\right)$, we conclude that for odd $n$ one has

$$
\begin{aligned}
S_{n}\left(\tau_{n}, \lambda\right) & =D_{n}(\lambda) C\left(\lambda^{2 n+1}\right) S\left(1, \lambda^{2 n+1}\right)^{-1} C\left(\lambda^{2 n+1}\right)^{-1} D_{n}(\lambda)^{-1} \\
& =C\left(\lambda^{n+1}\right) S\left(1, \lambda^{2 n+1}\right)^{-1} C\left(\lambda^{n+1}\right)^{-1} .
\end{aligned}
$$

Now we can express the characteristic function $r_{n}$ of $x_{n}$ by the characteristic function $r=r_{0}$ for the special symmetric solution $x$.

3.3. Theorem. Let $\lambda \in \boldsymbol{C} \backslash\{0\}$ and $n \in N_{0}$. Then

$$
r_{n}(\lambda)=1-\lambda+\frac{(-1)^{n}}{\lambda^{n}}\left[r\left(\lambda^{2 n+1}\right)+\lambda^{2 n+1}-1\right] .
$$


Proof. 1. Claim: $\operatorname{det} S(1, \mu)=1$ for $\mu \neq 0$. Indeed, since $x$ is odd, we have

$$
\begin{aligned}
a(1-t) & =f_{x}(x(1-t), y(1-t))=f_{x}(x(1-t), x(-t)) \\
& =f_{x}(-x(t-1),-x(t))=f_{x}(x(t-1), x(t)) \\
& =f_{x}(y(t), x(t))=-d(t)
\end{aligned}
$$

and hence $\int_{0}^{1}(a(t)+d(t)) d t=\int_{0}^{1}(a(t)-a(1-t)) d t=0$. The claim follows from $\operatorname{det} S(1, \mu)=\operatorname{det} S(0, \mu) \exp \left[\int_{0}^{1} \operatorname{tr} A(t, \mu) d t\right]=\exp \left[\int_{0}^{1}(a(t)+d(t)) d t\right]$.

2. Now let $\lambda \in C \backslash\{0\}$, set $\mu:=\lambda^{2 n+1}$ and set $S(1, \mu)=:\left(\begin{array}{ll}p & u \\ q & v\end{array}\right)$. Then $p v-q u=$ $\operatorname{det} S(1, \mu)=1$ and $S(1, \mu)^{-1}=\left(\begin{array}{cc}v & -u \\ -q & p\end{array}\right)$.

3. For even $n \in N_{0}$ we find, using Lemma 3.1 and Lemma 3.2,

$$
\begin{aligned}
r_{n}(\lambda) & =\operatorname{det}\left[S_{n}\left(\tau_{n}, \lambda\right)-C(\lambda)\right]=\operatorname{det}\left[D_{n}(\lambda) S(1, \mu) D_{n}(\lambda)^{-1}-C(\lambda)\right] \\
& =\operatorname{det}\left[\left(\begin{array}{cc}
1 & 0 \\
0 & \lambda^{n}
\end{array}\right)\left(\begin{array}{cc}
p & u \\
q & v
\end{array}\right)\left(\begin{array}{cc}
1 & 0 \\
0 & 1 / \lambda^{n}
\end{array}\right)-C(\lambda)\right] \\
& =\operatorname{det}\left[\left(\begin{array}{cc}
p & u \\
\lambda^{n} q & \lambda^{n} v
\end{array}\right)\left(\begin{array}{cc}
1 & 0 \\
0 & 1 / \lambda^{n}
\end{array}\right)-C(\lambda)\right] \\
& =\operatorname{det}\left[\left(\begin{array}{cc}
p & u / \lambda^{n} \\
\lambda^{n} q & v
\end{array}\right)-\left(\begin{array}{cc}
0 & \lambda \\
1 & 0
\end{array}\right)\right]=\operatorname{det}\left[\left(\begin{array}{cc}
p & u / \lambda^{n}-\lambda \\
\lambda^{n} q-1 & v
\end{array}\right)\right] \\
& =p v-\left(u / \lambda^{n}-\lambda\right)\left(\lambda^{n} q-1\right)=p v-u q+\lambda^{n+1} q+u / \lambda^{n}-\lambda \\
& =1+\left(\lambda^{2 n+1} q+u\right) / \lambda^{n}-\lambda=1-\lambda+(-1)^{n}(\mu q+u) / \lambda^{n} .
\end{aligned}
$$

4. For odd $n$, we derive

$$
\begin{aligned}
r_{n}(\lambda) & =\operatorname{det}\left[S_{n}\left(\tau_{n}, \lambda\right)-C(\lambda)\right]=\operatorname{det}\left[C\left(\lambda^{n+1}\right) S(1, \mu)^{-1} C\left(\lambda^{n+1}\right)^{-1}-C(\lambda)\right] \\
& =\operatorname{det}\left[\left(\begin{array}{cc}
0 & \lambda^{n+1} \\
1 & 0
\end{array}\right)\left(\begin{array}{cc}
v & -u \\
-q & p
\end{array}\right)\left(\begin{array}{cc}
0 & 1 \\
1 / \lambda^{n+1} & 0
\end{array}\right)-C(\lambda)\right] \\
& =\operatorname{det}\left[\left(\begin{array}{cc}
-\lambda^{n+1} q & \lambda^{n+1} p \\
v & -u
\end{array}\right)\left(\begin{array}{cc}
0 & 1 \\
1 / \lambda^{n+1} & 0
\end{array}\right)-C(\lambda)\right] \\
& =\operatorname{det}\left[\left(\begin{array}{cc}
p & -\lambda^{n+1} q \\
-u / \lambda^{n+1} & v
\end{array}\right)-\left(\begin{array}{ll}
0 & \lambda \\
1 & 0
\end{array}\right)\right] \\
& =\operatorname{det}\left[\left(\begin{array}{cc}
p & -\lambda^{n+1} q-\lambda \\
-u / \lambda^{n+1}-1 & v
\end{array}\right)\right] \\
& =p v-\left(\lambda^{n+1} q+\lambda\right)\left(u / \lambda^{n+1}+1\right)=p v-q u-u / \lambda^{n}-\lambda^{n+1} q-\lambda \\
& =1-\left(u+\lambda^{2 n+1} q\right) / \lambda^{n}-\lambda=1-\lambda+(-1)^{n}(u+\mu q) / \lambda^{n} .
\end{aligned}
$$

Thus we have $r_{n}(\lambda)=1-\lambda+(-1)^{n}(u+\mu q) / \lambda^{n}$ for all $n \in N_{0}$. Recall that $u=u(\mu), q=$ $q(\mu)$. We have, in particular, for $n=0$ and all $\mu \in C \backslash\{0\}$ that $r(\mu)=r_{0}(\mu)=1-\mu+$ 
$u(\mu)+\mu q(\mu)$, so $u(\mu)+\mu q(\mu)=r(\mu)+\mu-1$ for all $\mu \in C \backslash\{0\}$. For general $n \in N_{0}$, we hence obtain $r_{n}(\lambda)=1-\lambda+(-1)^{n} \lambda^{-n}\left[r\left(\lambda^{2 n+1}\right)+\lambda^{2 n+1}-1\right]$.

\section{Multipliers of rapidly and slowly oscillating solutions.}

4.1. Applications to stability/instability of rapidly oscillating periodic solutions. We use the notation of the previous section. Recall that 1 is always a trivial Floquet multiplier of $x_{n}$. We show that this implies that -1 is a trivial semi-Floquet multiplier of $x_{n}$, and hence $r_{n}(-1)=0$. This is well known for $n=0$, and it follows from $r(-1)=0$ and from Theorem 3.3 that

$$
\begin{aligned}
r_{n}(-1) & =1-(-1)+\frac{(-1)^{n}}{(-1)^{n}}\left[r\left((-1)^{2 n+1}\right)+(-1)^{2 n+1}-1\right] \\
& =1+1+[r(-1)+(-1)-1]=0 .
\end{aligned}
$$

It is also well-known that $\lim _{|\lambda| \rightarrow \infty} r(\lambda) / \lambda=-1$ (see, e.g., Lemma 2.2 in [7]). Slightly more can be shown: We have seen that $r(\lambda)=1-\lambda+u+\lambda q$, where $S(1, \lambda)=\left(\begin{array}{ll}p & u \\ q & v\end{array}\right)(1)$, and $\left(\begin{array}{l}\dot{p} \\ \dot{q}\end{array}\right)=\left(\begin{array}{c}a p+b q \\ \lambda^{-1} c p+d q\end{array}\right), \quad\left(\begin{array}{l}p(0) \\ q(0)\end{array}\right)=\left(\begin{array}{l}1 \\ 0\end{array}\right), \quad\left(\begin{array}{c}\dot{u} \\ \dot{v}\end{array}\right)=\left(\begin{array}{c}a u+b v \\ \lambda^{-1} c u+d v\end{array}\right), \quad\left(\begin{array}{l}u(0) \\ v(0)\end{array}\right)=\left(\begin{array}{l}0 \\ 1\end{array}\right)$.

For $|\lambda| \rightarrow \infty, u$ and $v$ converge to the solution of

$$
\left(\begin{array}{c}
\dot{u} \\
\dot{v}
\end{array}\right)=\left(\begin{array}{c}
a u+b v \\
d v
\end{array}\right), \quad\left(\begin{array}{l}
u(0) \\
v(0)
\end{array}\right)=\left(\begin{array}{l}
0 \\
1
\end{array}\right),
$$

uniformly on compact intervals. Hence $\lim _{|\lambda| \rightarrow \infty} u(1)=: u^{*}$ exists. If $\bar{q}:=\lambda q$, then

$$
\left(\begin{array}{c}
\dot{p} \\
\dot{\bar{q}}
\end{array}\right)=\left(\begin{array}{c}
a p+b q \\
c p+\lambda d q
\end{array}\right)=\left(\begin{array}{c}
a p+(b / \lambda) \bar{q} \\
c p+d \bar{q}
\end{array}\right), \quad\left(\begin{array}{l}
p(0) \\
\bar{q}(0)
\end{array}\right)=\left(\begin{array}{l}
1 \\
0
\end{array}\right),
$$

and again $p$ and $\bar{q}$ converge to the solution of

$$
\left(\begin{array}{c}
\dot{p} \\
\dot{\bar{q}}
\end{array}\right)=\left(\begin{array}{c}
a p \\
c p+d q
\end{array}\right), \quad\left(\begin{array}{c}
p(0) \\
\bar{q}(0)
\end{array}\right)=\left(\begin{array}{l}
1 \\
0
\end{array}\right),
$$

uniformly on compact intervals. Thus $\lim _{|\lambda| \rightarrow \infty} \lambda q(1)=: q^{*}$ exists.

We infer $\lim _{|\lambda| \rightarrow \infty}(r(\lambda)+\lambda)=1+u^{*}+q^{*}=: r^{*}$. As a consequence, we have

$$
\lim _{|\lambda| \rightarrow \infty}\left(r_{n}(\lambda)+\lambda\right)=\lim _{|\lambda| \rightarrow \infty}\left[1+\frac{(-1)^{n}}{\lambda^{n}}\left(r\left(\lambda^{2 n+1}\right)+\lambda^{2 n+1}-1\right)\right]=1 .
$$

It follows that, in particular, for $n \in N_{0}$ one has $\operatorname{sign} r_{n}(\lambda)=-\operatorname{sign}(\lambda)$ for all real $\lambda$ with sufficiently large absolute value. If we have some knowledge of $r_{n}$ on the real axis, then we can draw conclusions on instability of $x_{n}$. For example, if there is a real $\lambda<-1$ with $r_{n}(\lambda)<0$, then there is a real $\mu<\lambda$ with $r_{n}(\mu)=0$, and hence $x_{n}$ has a Floquet multiplier with norm $>1$ and therefore is unstable. On the other hand, if there is a real $\lambda>1$ with $r_{n}(\lambda)>0$, we can argue in the same way.

One way to prove $r_{n}(\lambda)<0$ for some $\lambda<-1$ is to look at the sign of $r_{n}^{\prime}(-1)$. Since $r_{n}(-1)=0$, the property $r_{n}^{\prime}(-1)>0$ implies the instability result. Let us calculate $r_{n}^{\prime}(-1)$. 
First we have

$$
\begin{aligned}
r_{n}^{\prime}(\lambda)= & -1+(-1)^{n}\left(\frac{-n}{\lambda^{n+1}}\right)\left[r\left(\lambda^{2 n+1}\right)+\lambda^{2 n+1}-1\right] \\
& +\frac{(-1)^{n}}{\lambda^{n}}\left[r^{\prime}\left(\lambda^{2 n+1}\right)+1\right](2 n+1) \lambda^{2 n},
\end{aligned}
$$

and thus

$$
\begin{aligned}
r_{n}^{\prime}(-1)= & -1+(-1)^{n}\left(\frac{-n}{(-1)^{n+1}}\right)\left[r\left((-1)^{2 n+1}\right)+(-1)^{2 n+1}-1\right] \\
& +\frac{(-1)^{n}}{(-1)^{n}}\left[r^{\prime}\left((-1)^{2 n+1}\right)+1\right](2 n+1)(-1)^{2 n} \\
= & -1+n[r(-1)+(-1)-1]+\left[r^{\prime}(-1)+1\right](2 n+1) \\
= & -1-2 n+(2 n+1)\left(r^{\prime}(-1)+1\right)=(2 n+1) r^{\prime}(-1)
\end{aligned}
$$

Therefore $r_{n}^{\prime}(-1)$ and $r^{\prime}(-1)$ have the same sign and we conclude:

If $r^{\prime}(-1)>0$, then every solution $x_{n}$ of equation $\left(f_{n}\right)\left(n \in N_{0}\right)$ is unstable.

EXAMPLE. Consider the case $f(x, y)=g(x) \cdot h(y)$, where $g$ is even, and $h$ is odd. The feedback condition from the beginning of Section 2 is fulfilled if $g(0) \neq 0$ and $h^{\prime}(0) \neq 0$. In [7], Thm. 3.1, it was shown that $r^{\prime}(-1)>0$, provided that $h^{\prime}$ is decreasing on $[0, \infty)$ and $g$ is increasing on $[0, \infty)$. Therefore all solutions $x_{n}$ are unstable in this case.

Another way to conclude $r_{n}(\lambda)<0$ for some $\lambda<-1$ is to reduce this problem to the question $r(\lambda)<0, \lambda<-1$. To this end we observe that

$$
\begin{aligned}
(-\lambda)^{n} r_{n}(\lambda) & =(-1)^{n} \lambda^{n} r_{n}(\lambda) \\
& =(-1)^{n} \lambda^{n}\left[1-\lambda+\frac{(-1)^{n}}{\lambda^{n}}\left(r\left(\lambda^{2 n+1}\right)+\lambda^{2 n+1}-1\right)\right] \\
& =(-1)^{n} \lambda^{n}-(-1)^{n} \lambda^{n+1}+r\left(\lambda^{2 n+1}\right)+\lambda^{2 n+1}-1 \\
& =r\left(\lambda^{2 n+1}\right)+\lambda^{2 n+1}-(-1)^{n} \lambda^{n+1}+(-1)^{n} \lambda^{n}-1 \\
& =r\left(\lambda^{2 n+1}\right)+\left((-1)^{n} \lambda^{n+1}+1\right)\left((-1)^{n} \lambda^{n}-1\right) \\
& =r\left(\lambda^{2 n+1}\right)-\left((-\lambda)^{n+1}-1\right)\left((-\lambda)^{n}-1\right) .
\end{aligned}
$$

Now assume that there is a real $\mu<-1$ with $r(\mu) \leq 0$ (which implies existence of a semiFloquet multiplier of $x$ in $(-\infty, \mu])$. Let $\lambda<-1$ be such that $\lambda^{2 n+1}=\mu$. Then $-\lambda>1$ and $(-\lambda)^{k}>1$ for all $k \in N_{0}$, and

$$
(-\lambda)^{n} r_{n}(\lambda)=r(\mu)-\left((-\lambda)^{n+1}-1\right)\left((-\lambda)^{n}-1\right)<0
$$

yields $r_{n}(\lambda)<0$. We can conclude:

If $x$ has a real semi-Floquet multiplier less than -1 , then all $x_{n}\left(n \in N_{0}\right)$ are unstable. 
Next assume that there is a real $\mu>1$ with $r(\mu) \geq 0$ (which implies existence of a semi-Floquet multiplier of $x$ in $[\mu, \infty)$ ). Let $\lambda>1$ be such that $\lambda^{2 n+1}=\mu$. If $n$ is even, then

$$
\begin{aligned}
\lambda^{n} r_{n}(\lambda) & =(-\lambda)^{n} r_{n}(\lambda)=r(\mu)-\left((-\lambda)^{n+1}-1\right)\left((-\lambda)^{n}-1\right) \\
& =r(\mu)-\left(-\lambda^{n+1}-1\right)\left(\lambda^{n}-1\right) \\
& =r(\mu)+\left(\lambda^{n+1}+1\right)\left(\lambda^{n}-1\right)>0,
\end{aligned}
$$

which implies $r_{n}(\lambda)>0$. Hence we conclude:

If $x$ has a real semi-Floquet multiplier greater than 1 , then $x_{n}$ is unstable for even $n \in N$.

Unfortunately, no conclusion can be made for odd $n$, since then

$$
\begin{aligned}
-\lambda^{n} r_{n}(\lambda) & =(-\lambda)^{n} r_{n}(\lambda)=r(\mu)-\left((-\lambda)^{n+1}-1\right)\left((-\lambda)^{n}-1\right) \\
& =r(\mu)-\left(\lambda^{n+1}-1\right)\left(-\lambda^{n}-1\right) \\
& =r(\mu)+\left(\lambda^{n+1}-1\right)\left(\lambda^{n}+1\right)>0,
\end{aligned}
$$

which only implies $r_{n}(\lambda)<0$.

More generally we expect that if $r(\mu)=0$ for some $\mu \in \boldsymbol{C}$ with $|\mu|>1$, then $r_{n}(\lambda)=0$ for some $\lambda \in C$ with $|\lambda|>1$. The reason for this expectation is that, in terms of general experience, rapidly oscillating solutions are less stable than slowly oscillating ones. Hence, intuitively one does not expect an increase of stability from a transformation of slowly oscillating to rapidly oscillating periodic solutions. However, we have no proof for this intuitive conjecture.

4.2. Application to secondary bifurcation. Let us now study the equation $\dot{x}(t)=$ $f(x(t), x(t-\alpha))$, or, equivalently,

$$
\dot{x}(t)=\alpha f(x(t), x(t-1))
$$

with delay parameter $\alpha \in \boldsymbol{R}$. Conditions on $f$ which imply the existence of a primary branch PB of special symmetric periodic solutions can be found, e.g., in [6, 12]. Assuming $\gamma=$ $f_{y}(0,0) \neq 0$, the primary branch PB bifurcates at $\alpha=-(\pi / 2 \gamma)$ from the zero solution. PB is a smooth curve in the space $\boldsymbol{R} \times C$ in a neighborhood of $(-(\pi / 2 \gamma), 0)$. More specifically, there exists $\delta>0$ (depending on the nonlinearity $f$ ), and a smooth function $[0, \delta) \ni z \mapsto$ $\left(\alpha(z), \varphi_{z}\right) \in \boldsymbol{R} \times C$ such that for each $z \in[0, \delta)$, the above equation with parameter $\alpha(z)$ has a SSPS with amplitude (maximal value) $z$ and initial value $\varphi_{z}$. One has $\alpha(0)=-(\pi / 2 \gamma), \varphi_{0}=$ 0 and $\varphi_{z}(-1)=0, \varphi_{z}(\cdot)>0$ on $(-1,0]$ and $\varphi_{z}(0)=z$ for all $z \in(0, \delta)$. The branch is best visualized as the graph of the function $z \mapsto \alpha(z)$ (see also [8]).

Applying Cooke's and Saupe's transformations to all solutions that correspond to points on $\mathrm{PB}$, we get primary branches $\mathrm{PB}_{n}$ of symmetric solutions with periods $4 /(2 n+1)$. Clearly, $\mathrm{PB}_{n}$ bifurcates at $\alpha=-(-1)^{n}(2 n+1) \pi /(2 \gamma)$ from the zero solution. Assume that for some $k \in N$ we have a period-times- $k$ bifurcation on PB in the following sense: We have a secondary branch $S$ which is a curve $w \mapsto\left(\tilde{\alpha}(w), \psi_{w}\right) \in \boldsymbol{R} \times C$ defined on some interval containing zero, and such that $\tilde{\alpha}(0)=\alpha(z), \psi_{0}=\varphi_{z}$ for some $z \in[0, \delta)$. Furthermore, for all $w \neq 0$, we have that $\left(\tilde{\alpha}(w), \psi_{w}\right)$ does not lie on $\mathrm{PB}$, and the solution of the above equation with parameter $\tilde{\alpha}(w)$ and initial value $\psi_{w}$ is periodic with minimal period approximately 
equal to $k \cdot 4$. In case $k>1$ this is a subharmonic bifurcation, for example, a period doubling. Let us write $k \odot$ PB for short.

Now we apply Saupe's transformations $\mathcal{S}_{n}$ to the solutions which correspond to points on $S$. In general such solutions (with minimal period $\omega$ ) are nonsymmetric in the sense that they do not have the symmetry property $x(t+\omega / 2)=-x(t)(t \in \boldsymbol{R})$. Hence we must take $n$ even and thus use Cooke's transformation $\mathcal{C}_{n}$ (compare the remark at the end of Section 2). We get a branch $\mathcal{C}_{n} S$, and the corresponding solutions have periods close to $(2 \cdot 4 k) /(n \cdot 4 k+2)=$ $4 k /(2 k n+1)$. Let now $x: \boldsymbol{R} \rightarrow \boldsymbol{R}$ be the solution of the above equation with parameter $\tilde{\alpha}(0)$ and initial value $\psi_{0}$ (which corresponds to the intersection point of $S$ and PB). Then $x$ is transformed to $\tilde{x}(t)=x((1+(n / 2) \cdot 4 k) t)=x((1+2 n k) t)=x_{n k}(t)$ (compare the end of Section 2). I.e., $\tilde{x}$ corresponds to a point on $\mathcal{C}_{n} S$ and on $\mathrm{PB}_{n k}$. Hence we have secondary bifurcation on $\mathrm{PB}_{n k}$, or for short

$$
k \odot \mathrm{PB} \Rightarrow k \odot \mathrm{PB}_{n k} \text { via } \mathcal{C}_{n} \text { for even } n \in N .
$$

EXAMPLE. Assume we have a symmetry breaking secondary bifurcation of PB which is not subharmonic. That is, a secondary branch $S$ as above, with corresponding nonsymmetric solutions of minimal period $\omega$ approximately 4. This corresponds to the case $k=1$ in the above formulas. Then we also have symmetry breaking (and not subharmonic) bifurcations on $\mathrm{PB}_{2}, \mathrm{~PB}_{4}, \ldots$ If we have a period doubling bifurcation on $\mathrm{PB}$, i.e., $k=2$, then we have this kind of bifurcation on $\mathrm{PB}_{4}, \mathrm{~PB}_{8}, \ldots$ as well.

Let us see how these observations are reflected in the formula for $r_{n}$. The property $k \odot \mathrm{PB}$ indicates that some $x^{\alpha} \in \mathrm{PB}$ has a Floquet multiplier $\mu$ with $\mu^{k}=1$. We want to show that $x_{n k}^{\alpha}, n$ even has the same property. To this end, let $r$ be the characteristic function of $x^{\alpha}$. Since $\mu$ is a Floquet multiplier, there is a semi-Floquet multiplier $\lambda$ with $\mu=\lambda^{2}$. Since $n$ is even, we see that $\lambda^{n k}=\left(\lambda^{2 k}\right)^{n / 2}=\left(\mu^{k}\right)^{n / 2}=1$ and $\lambda^{2 n k+1}=\lambda$. Now $r(\lambda)=0$ implies

$$
\begin{aligned}
r_{n k}(\lambda) & =1-\lambda+\frac{(-1)^{n k}}{\lambda^{n k}}\left[r\left(\lambda^{2 n k+1}\right)+\lambda^{2 n k+1}-1\right] \\
& =1-\lambda+1 \cdot[r(\lambda)+\lambda-1]=0 .
\end{aligned}
$$

Hence $\lambda$ is also a semi-Floquet multiplier of $x_{n k}^{\alpha}$, and $\lambda^{2}=\mu$ is also a Floquet multiplier of $x_{n k}^{\alpha}$.

We can treat the case of odd $n$ in the following way. Observe that if $x^{\alpha} \in \mathrm{PB}$, then $x_{n}^{\alpha}=-x_{-n-1}^{\alpha}$. Let $\mathcal{C}_{n}^{\prime}:=-\mathcal{C}_{n-1}$, i.e., if $x$ has period $\omega$, then $\mathcal{C}_{n}^{\prime} x$ is the function $\boldsymbol{R} \ni t \mapsto$ $-x((1-((n+1) / 2) \omega) t) \in \boldsymbol{R}$, which has period $2 \omega /((n+1) \omega-2)$. If we apply $\mathcal{C}_{n}^{\prime}$ to the special symmetric solutions of $\mathrm{PB}$, we get $\mathrm{PB}_{n}$. Since $\mathcal{C}_{n}^{\prime}=-\mathcal{C}_{-n-1}$ and since $-n-1$ is even if $n$ is odd, we can apply $\mathcal{C}_{n}^{\prime}$ to nonsymmetric solutions in this case. If we have a solution $x^{\alpha}$ corresponding to a bifurcation point on $\mathrm{PB}$ with periods close to $4 k$, then $\mathcal{C}_{n}^{\prime}$ yields solutions with periods close to $2 \cdot 4 k /((n+1) \cdot 4 k-2)=k \cdot 4 /(2 k n+2 k-1) \cdot x^{\alpha}$ is mapped to $x_{k n+k-1}^{\alpha}$, and hence we have secondary bifurcation on $\mathrm{PB}_{k n+k-1}$; for short:

$$
k \odot \mathrm{PB} \Rightarrow k \odot \mathrm{PB}_{n k+k-1} \text { via } \mathcal{C}_{n}^{\prime} \text { for odd } n .
$$


Let us show again that this result is in accordance with our formula for $r_{n}$. Let $\mu=\lambda^{2}$ be a Floquet multiplier of $x^{\alpha} \in \mathrm{PB}$ with $\mu^{k}=1$. Then $n k+k=(n+1) k$ is even and $\lambda^{n k+k}=\left(\lambda^{2 k}\right)^{(n+1) / 2}=1, \lambda^{2 n k+2 k}=1$. It follows that

$$
\begin{aligned}
r_{n k+k-1}(1 / \lambda) & =1-(1 / \lambda)+(-\lambda)^{n k+k-1}\left[r\left(1 / \lambda^{2 n k+2 k-1}\right)+1 / \lambda^{2 n k+2 k-1}-1\right] \\
& =1-1 / \lambda+(-1 / \lambda)[r(\lambda)+\lambda-1] \\
& =1-1 / \lambda-r(\lambda) / \lambda-1+1 / \lambda=-r(\lambda) / \lambda=0 .
\end{aligned}
$$

Hence $\tilde{\lambda}=1 / \lambda$ is a semi-Floquet multiplier and $\tilde{\mu}=1 / \lambda^{2}=1 / \mu$ is a Floquet multiplier of $x_{n k+k-1}$, and of course $\tilde{\mu}^{k}=1 / \mu^{k}=1$. Combining both results, we arrive at

$$
k \odot \mathrm{PB} \Rightarrow k \odot \mathrm{PB}_{m} \text { for } m=2 k, 4 k, 6 k, \ldots \text { and } m=2 k-1,4 k-1, \ldots
$$

For example, symmetry (not subharmonic) breaking bifurcation on PB implies symmetry breaking bifurcation on $\mathrm{PB}_{1}, \mathrm{~PB}_{2}, \ldots$, while period doubling bifurcation on $\mathrm{PB}$ implies period doubling bifurcation on $\mathrm{PB}_{3}, \mathrm{~PB}_{4}, \mathrm{~PB}_{7}, \mathrm{~PB}_{8}, \mathrm{~PB}_{11}, \mathrm{~PB}_{12}, \ldots$.

In the same way one can study secondary bifurcation on $\mathrm{PB}_{m}$. One finds

$$
\begin{aligned}
& k \odot \mathrm{PB}_{m} \Rightarrow k \odot \mathrm{PB}_{n k+m} \text { via } \mathcal{C}_{n} \text { for even } n, \\
& k \odot \mathrm{PB}_{m} \Rightarrow k \odot \mathrm{PB}_{n k+k-m-1} \text { via } \mathcal{C}_{n}^{\prime} \text { for odd } n,
\end{aligned}
$$

or, combined,

$$
k \odot \mathrm{PB}_{m} \Rightarrow k \odot \mathrm{PB}_{n} \text { if } n-m \text { or } n+m+1 \text { is a multiple of } 2 k .
$$

As a consequence, we have that we know all period-times- $k$ bifurcations on $\mathrm{PB}_{n}, n \in N_{0}$, if we know them on $\mathrm{PB}_{0}, \ldots, \mathrm{PB}_{k-1}$. Namely, if $k \odot \mathrm{PB}_{m}$ for some $m \in N_{0}$, then there is an $n \in\{0,1, \ldots, k-1\}$ with $k \odot \mathrm{PB}_{n}$ since we can always write $m=2 k \cdot q+n$ for some $n \in\{0,1, \ldots, 2 k-1\}$. Now $n-m=-2 k q$ and $(2 k-1-n)+m+1=2 k(q+1)$ are multiples of $2 k$, i.e., $k \odot \mathrm{PB}_{n}$ and $k \odot \mathrm{PB}_{2 k-1-n}$. But either $n \in\{0,1, \ldots, k-1\}$ or $(2 k-1-n) \in\{0,1, \ldots, k-1\}$.

EXAMPLE. It is sufficient to study symmetry breaking (not subharmonic) bifurcation $(k=1)$ on $\mathrm{PB}$ and period doubling bifurcation $(k=2)$ on $\mathrm{PB}$ and $\mathrm{PB}_{1}$; all such bifurcations on $\mathrm{PB}_{n}$ are just transforms of these bifurcation points.

4.3. Asymptotic behavior of semi-Floquet multipliers. In [4], the asymptotic behavior of semi-Floquet multipliers of special symmetric solutions of

$$
\dot{x}(t)=-\alpha f(x(t-1))
$$

was studied for sine-like functions $f$ (see Thm. 1 in [4]) as $\alpha \rightarrow \infty$. The key for the result given there is that if $\kappa(\lambda):=(1 / 2)|\operatorname{Im}(1 / \sqrt{\lambda})| \leq 1 / 2$, then

$$
\left|r^{\alpha}(\lambda)+\lambda-1\right| \leq c \alpha e^{\alpha(2 \kappa(\lambda)-1)}
$$

for some constant $c$; here $r^{\alpha}$ is the characteristic function of the Kaplan-Yorke-solution $x^{\alpha}$ for the parameter $\alpha$. Assume that there is a sequence $\alpha_{k} \rightarrow \infty$ with semi-Floquet multipliers $\lambda_{k}$ 
such that $\kappa\left(\lambda_{k}\right) \leq 1 / 2-\gamma$ for some $\gamma>0$. Then

$$
\left|\lambda_{k}-1\right|=\left|r^{\alpha_{k}}\left(\lambda_{k}\right)+\lambda_{k}-1\right| \leq c \alpha_{k} e^{\alpha_{k}\left(2 \kappa\left(\lambda_{k}\right)-1\right)} \leq c \alpha_{k} e^{-\gamma \alpha_{k}},
$$

which shows $\lambda_{k} \rightarrow 1$. Hence we have for $\alpha \rightarrow \infty$ that, for every semi-Floquet multiplier $\lambda$ of $x^{\alpha}$,

- either $\lambda$ is close to 1 ,

- or $\kappa(\lambda)$ is close to or greater than $1 / 2$, i.e., $|\operatorname{Im}(1 / \sqrt{\lambda})|$ is close to or greater than 1 .

Since $|\operatorname{Im}(1 / \sqrt{\lambda})|=1$ defines the so-called cardioide curve, the semi-Floquet multipliers of $x^{\alpha}$ are close to the heart-shaped area

$$
\mathcal{H}=\{\lambda \in \boldsymbol{C} \backslash\{0\}|| \operatorname{Im}(1 / \sqrt{\lambda}) \mid \geq 1\} \cup\{0,1\} \subset \boldsymbol{C} .
$$

We can immediately show an analogous result for the semi-Floquet multipliers of $x_{n}^{\alpha}$ on the transformed primary branch $\mathrm{PB}_{n}$. It is convenient to say that $\mu \in \boldsymbol{C}$ is a $(2 n+1) / 2$-Floquet multiplier of $x_{n}^{\alpha}$, if there is a semi-Floquet multiplier $\lambda \in \boldsymbol{C}$ of $x_{n}^{\alpha}$ with $\mu=\lambda^{2 n+1}$. Then

$$
\left|r_{n}^{\alpha}(\lambda)+\lambda-1\right|=\left|\frac{(-1)^{n}}{\lambda^{n}}\left[r^{\alpha}(\mu)+\mu-1\right]\right| \leq \frac{1}{|\lambda|^{n}} c \alpha e^{\alpha(2 \kappa(\mu)-1)} .
$$

Now we can apply the same argument as above: Assume that we have a sequence $\alpha_{k} \rightarrow$ $\infty$ with $(2 n+1) / 2$-Floquet multipliers $\mu_{k}=\lambda_{k}^{2 n+1}$ of $x_{n}^{\alpha_{k}}$ such that

$$
\kappa\left(\mu_{k}\right) \leq \frac{1}{2}-\gamma \quad \text { and } \quad\left|\mu_{k}\right| \geq \beta
$$

for some constants $\beta, \gamma>0$. Then $\left|\lambda_{k}\right| \geq \beta^{1 /(2 n+1)}$, and the estimate

$$
\left|\lambda_{k}-1\right|=\left|r_{n}^{\alpha_{k}}\left(\lambda_{k}\right)+\lambda_{k}-1\right| \leq \frac{1}{\left|\lambda_{k}\right|^{n}} c e^{\alpha_{k}\left(2 \kappa\left(\mu_{k}\right)-1\right)} \leq \frac{1}{\beta^{n /(2 n+1)}} c e^{-2 \alpha_{k} \gamma}
$$

shows that $\lambda_{k} \rightarrow 1$ and $\mu_{k} \rightarrow 1$. Hence we have for $\alpha \rightarrow \infty$ and for $(2 n+1) / 2$-Floquet multipliers $\lambda$ of $x_{n}^{\alpha}$ that

- either $\lambda$ is close to 1 ,

- or $\lambda$ is close to 0 ,

- or $\lambda$ is close to the set $\{\lambda \in \boldsymbol{C}|| \operatorname{Im}(1 / \sqrt{\lambda}) \mid \geq 1\}$.

Because of $0,1 \in \mathcal{H}$, the $(2 n+1) / 2$-Floquet multipliers are in any case close to $\mathcal{H}$.

In the next section we give a simple example of a sine-like function $f$, for which $r(\lambda)$ can be calculated explicitly. This will allow us to obtain detailed information on the semi-Floquet multipliers on $\mathrm{PB}_{n}$.

5. A specific example with piecewise linear feedback. We study a piecewise linear model, where the characteristic function $r=r_{0}$ of the untransformed solutions is known explicitly, and Theorem 3.3 enables us to obtain information on the multipliers of transformed, rapidly oscillating solutions. Set $f(x):=1 / 2-|x-1 / 2|$ for $x \in[0,1]$, and continue $f$ to an odd and two-periodic function, which then is a piecewise linear caricature of $x \mapsto$ $(1 / \pi) \sin (\pi x)$.

We know that for $z \in(1 / 2,1)$ there is an $\alpha_{z}>\pi / 2$ such that the equation

$$
\left(-\alpha_{z} f\right) \quad \dot{x}(t)=-\alpha_{z} f(x(t-1))
$$


has a special symmetric solution $x_{z}$ with amplitude $z$. Let $r_{z}$ be its characteristic function. In Section 7 of [5] the following expressions for $\alpha_{z}$ and $r_{z}$ were calculated: If $\mu$ is such that $\lambda \mu^{2}=1$, then

$$
\alpha_{z}=2 \tau(z)+2 \sigma(z), \quad r_{z}(\lambda)=1-\lambda-\frac{2}{\mu} s(z, \lambda) \cdot c(z, \lambda),
$$

where $\tau(z)=\pi / 4-\arctan \left(2 \sqrt{1 / 4-(1-z)^{2}}\right), \sigma(z)=\operatorname{Arcosh}[1 /(2(1-z))]$, and

$$
s(z, \lambda)=\sinh (2 \mu \tau(z)), \quad c(z, \lambda)=\cos (2 \mu \sigma(z)) .
$$

For the remainder of this section, we now fix $n \in N$, and set $\tau_{n}:=1 /(2 n+1)$. As in Section 1 , the function $x_{z, n}$ defined by $x_{z, n}(t)=x_{z}((2 n+1) t)(t \in \boldsymbol{R})$ is a symmetric solution of the equation

$$
\left((-1)^{n+1}(2 n+1) \alpha_{z} f\right) \quad \dot{x}(t)=(-1)^{n+1}(2 n+1) \alpha_{z} f(x(t-1)),
$$

with period $4 /(2 n+1)$ and amplitude $z$. (Observe that if $n$ is odd, then this equation has positive feedback around zero.) Let $T=4 /(2 n+1)$ be the period of $x_{z, n}$. Note that, although $f$ is not everywhere differentiable, the time- $T$-map $\Phi(T, \cdot): C \rightarrow C$ of the semiflow generated by the above equation is still differentiable with respect to the initial value. It can be seen, e.g., from Lemma 6.5 in [16], that the derivative $D_{2} \Phi\left(T,\left(x_{z, n}\right)_{0}\right)$ (i.e., the monodromy operator) is still given by solutions of the variational equation, which now is a linear equation with piecewise constant coefficient. The characterization of semi-multipliers as zeroes of a determinant involving the fundamental solution matrix (now for a system with piecewise constant coefficients) from Lemma 3.1. remains valid, as well as the further results of Section 3, in particular, Theorem 3.3.

Let us now study the semi-multipliers of $x_{z, n}$. We know from Theorem 3.3 that these are the zeroes of the function $r_{z, n}$ given by

$$
r_{z, n}(\lambda)=1-\lambda+\frac{(-1)^{n}}{\lambda^{n}}\left[r_{z}\left(\lambda^{2 n+1}\right)+\lambda^{2 n+1}-1\right] .
$$

Using the above expression for $r_{z}$, we obtain that, if $\mu$ is such that

$$
\lambda^{2 n+1} \mu^{2}=1
$$

the expression in the bracket equals

$$
1-\lambda^{2 n+1}-\frac{2}{\mu} \sinh (2 \mu \tau(z)) \cos (2 \mu \sigma(z))+\lambda^{2 n+1}-1=-\frac{2}{\mu} \sinh (2 \mu \tau(z)) \cos (2 \mu \sigma(z)),
$$

so we conclude $r_{z, n}(\lambda)=1-\lambda+\left[(-1)^{n+1} 2 /\left(\lambda^{n} \mu\right)\right] \sinh (2 \mu \tau(z)) \cos (2 \mu \sigma(z))$.

For positive, real $\lambda$ we can set

$$
\mu:=\lambda^{-(2 n+1) / 2}, \quad \text { so } \quad \lambda=\mu^{-2 /(2 n+1)},
$$

and thus

$$
r_{z, n}(\lambda)=1-\mu^{-2 /(2 n+1)}+\left[(-1)^{n+1} 2 /\left(\mu^{1 /(2 n+1)}\right)\right] \sinh (2 \mu \tau(z)) \cos (2 \mu \sigma(z)) .
$$

We can draw a first conclusion from these expressions: 
5.1. Proposition. For $z \in(1 / 2,1)$, every positive real semi-Floquet multiplier $\lambda$ of $x_{z, n}$ satisfies

$$
\lambda \leq(e+1)^{2} .
$$

Proof. Note that for $\mu \in(0,1 / 2]$ and $z \in(1 / 2,1)$ one has

$$
\tau(z)=\pi / 4-\arctan \left(2 \sqrt{1 / 4-(1-z)^{2}}\right) \in(0, \pi / 4),
$$

and $|\sinh (2 \mu \tau(z)) \cos (\cdots)| \leq \max _{u \in[0,1]} \sinh (u) \leq e / 2$. If $\lambda \geq 2$, then $\mu:=\lambda^{-(2 n+1) / 2} \leq$ $1 / 2$, so the last estimate is valid. Observing that $\lambda^{n} \mu=\lambda^{-1 / 2}$, one sees that

$$
\left|r_{z, n}(\lambda)\right| \geq \lambda-1-2 \lambda^{1 / 2} e / 2=\lambda-1-\lambda^{1 / 2} e=\lambda^{1 / 2}\left(\lambda^{1 / 2}-e\right)-1 .
$$

Thus, if $\lambda \geq(e+1)^{2}$, then $\left|r_{z, n}(\lambda)\right| \geq e+1-1=e>0$. The assertion follows.

REMARK. Note that $\alpha_{z} \rightarrow \infty$ as $z \rightarrow 1$, so for odd $n$ the linearization of equation $\left((-1)^{n+1}(2 n+1) \alpha_{z} f\right)$ at zero has solutions $\exp (\lambda t)$ with a positive number $\lambda$ going to infinity as $z \rightarrow 1$. Consequently, the time $4 /(2 n+1)$ solution operator of the linearization at zero has unbounded positive eigenvalues as $z \rightarrow 1$. Proposition 5.1 shows that this is not the case for the monodromy operator of the $4 /(2 n+1)$ - periodic solution $x_{z, n}$, which oscillates about zero.

We are now interested in $z$ close to 1 , and in positive real multipliers. For $\mu>0$ and $\zeta \in(0,1 / 4)$ we set

$$
\psi(\mu, \zeta):=\sinh \left[2 \mu\left(\pi / 4-\arctan \left(2 \sqrt{1 / 4-\zeta^{2}}\right)\right)\right] \cdot \cos [2 \mu \operatorname{Arcosh}(1 /(2 \zeta))] .
$$

If $z \in(3 / 4,1), \lambda>0$, and $\mu:=\lambda^{-(2 n+1) / 2}, \zeta:=1-z$, we then have

$$
\mu^{2 \tau_{n}} r_{z, n}(\lambda)=\mu^{2 \tau_{n}}+(-1)^{n+1} 2 \mu^{\tau_{n}} \psi(\mu, \zeta)-1 .
$$

We define

$$
G_{n}:(0, \infty) \times(0,1 / 4) \rightarrow \boldsymbol{R}, \quad G_{n}(\mu, \zeta):=\mu^{2 \tau_{n}}+(-1)^{n+1} 2 \mu^{\tau_{n}} \psi(\mu, \zeta)-1 .
$$

Then, for $z \in(3 / 4,1)$, and $\lambda>0$, and $\mu, \zeta$ as above, we have $\mu^{2 \tau_{n}} r_{z, n}(\lambda)=G_{n}(\mu, 1-z)$. We now approximate the function $G_{n}$, expanding in powers of $\zeta$.

5.2. LEMMA. a) $G_{n}(\mu, \zeta)=\mu^{2 \tau_{n}}\left\{1+(-1)^{n+1} 4 \mu^{2 n \tau_{n}} \zeta^{2}[\cos (2 \mu \log \zeta)+R(\mu, \zeta)]\right\}$ -1 , where for $\mu \in(0,2)$ and $\zeta \in(0,1 / 4)$ one has $|R(\mu, \zeta)| \leq 28 \zeta^{2}$.

b) The function $\tilde{G}_{n}:(0,2) \times(-\infty, 1 / 4) \rightarrow \boldsymbol{R}$ defined by

$$
\tilde{G}_{n}(\mu, \zeta):= \begin{cases}G_{n}(\mu, \zeta) & \text { if } \zeta \in(0,1 / 4) \\ \mu^{2 \tau_{n}}-1 & \text { if } \zeta \leq 0\end{cases}
$$

is a $C^{1}$ extension of $G_{n}$.

Proof. a) Set $w(\zeta):=2 \sqrt{1 / 4-\zeta^{2}}$ and $\varphi(\zeta):=\pi / 4-\arctan (w(\zeta))$. Then

$$
\begin{aligned}
& \psi(\mu, \zeta)=\sinh (2 \mu \varphi(\zeta)) \cdot \cos [2 \mu \operatorname{Arcosh}(1 / 2 \zeta)], \quad \text { and } \\
& w(\zeta)=\sqrt{1-(2 \zeta)^{2}}=1-\frac{(2 \zeta)^{2}}{2}+R_{1}(\zeta)=1-2 \zeta^{2}+R_{1}(\zeta),
\end{aligned}
$$


where, using $\zeta \leq 1 / 4$, we get the estimate

$$
\left|R_{1}(\zeta)\right| \leq \frac{1}{2} \max _{u \in[3 / 4,1]}\left|\frac{d^{2}}{d x^{2}}(x \mapsto \sqrt{x})(u)\right| \cdot(2 \zeta)^{4} \leq \frac{1}{2} \frac{1}{4}\left(\frac{3}{4}\right)^{-3 / 2} 16 \zeta^{4}=2 \frac{2^{3}}{3^{3 / 2}} \zeta^{4} \leq 4 \zeta^{4} .
$$

Next, we have

$$
\varphi(\zeta)=\arctan ^{\prime}(1)(1-w(\zeta))+R_{2}(\zeta)
$$

where $\left|R_{2}(\zeta)\right| \leq(1 / 2) \max _{u \in[w(\zeta), 1]}\left|\arctan ^{\prime \prime}(u)\right| \cdot(1-w(\zeta))^{2}$. With

$$
\left|\arctan ^{\prime \prime}(u)\right|=\left|-2 u /\left(1+u^{2}\right)^{2}\right| \leq 1
$$

we get $\left|R_{2}(\zeta)\right| \leq(1 / 2)\left(2 \zeta^{2}+4 \zeta^{4}\right)^{2} \leq(1 / 2)\left(3 \zeta^{2}\right)^{2} \leq 5 \zeta^{4}$.

Combining (5.2.2) and (5.2.3), we can write

$$
\varphi(\zeta)=\frac{1}{2}\left(2 \zeta^{2}-R_{1}(\zeta)\right)+R_{2}(\zeta)=\zeta^{2}+R_{3}(\zeta), \quad \text { where } \quad\left|R_{3}(\zeta)\right| \leq 2 \zeta^{4}+5 \zeta^{4}=7 \zeta^{4}
$$

We now approximate the two factors of $\psi$ : First, $\sinh (2 \mu \varphi(\zeta))=2 \mu \varphi(\zeta)+R_{4}(\mu, \zeta)$, where for $\mu \in(0,2)$ and $\zeta \in(0,1 / 4)$ one has $|2 \mu \varphi(\zeta)| \leq 4\left(\zeta^{2}+7 \zeta^{4}\right) \leq 8 \zeta^{2} \leq 1 / 2$, and

$$
\begin{aligned}
\left|R_{4}(\mu, \zeta)\right| & \leq \frac{1}{6} \max _{u \in[0,1 / 2]}\left|\sinh ^{\prime \prime \prime}(u)\right|(2 \mu \varphi(\zeta))^{3} \leq \frac{1}{6} \frac{e^{1 / 2}}{2} 8 \mu^{3}\left(\zeta^{2}+7 \zeta^{4}\right)^{3} \\
& \leq \frac{8}{6} \mu^{3} \zeta^{6}\left(1+7 \zeta^{2}\right)^{3} \leq \frac{8}{6}(3 / 2)^{3} \mu^{3} \zeta^{6} \leq 5 \mu^{3} \zeta^{6}
\end{aligned}
$$

Hence $\sinh (2 \mu \varphi(\zeta))=2 \mu \zeta^{2}+R_{5}(\mu, \zeta)$, where for $\mu \in(0,2]$ and $\zeta \in(0,1 / 4]$

$$
\left|R_{5}(\mu, \zeta)\right|=\left|2 \mu R_{3}(\zeta)+R_{4}(\mu, \zeta)\right| \leq 14 \mu \zeta^{4}+5 \mu^{3} \zeta^{6} \leq 14 \mu \zeta^{4}+\frac{20}{16} \mu \zeta^{4} \leq 16 \mu \zeta^{4}
$$

Second, note that $(1 / 2 \zeta) \geq \sqrt{1 /\left(4 \zeta^{2}\right)-1}=(1 / 2 \zeta) \sqrt{1-4 \zeta^{2}} \geq(1 / 2 \zeta)\left(1-4 \zeta^{2}\right)=$ $(1 / 2 \zeta)-2 \zeta$, so we have $1 / \zeta \geq 1 / 2 \zeta+\sqrt{1 /\left(4 \zeta^{2}\right)-1} \geq 1 / \zeta-2 \zeta$. Consequently,

$$
\begin{aligned}
\operatorname{Arcosh}(1 / 2 \zeta) & =\log \left(1 / 2 \zeta+\sqrt{1 /\left(4 \zeta^{2}\right)-1}\right) \in[\log (1 / \zeta-2 \zeta), \log (1 / \zeta)], \quad \text { and } \\
\operatorname{Arcosh}(1 / 2 \zeta) & \geq \log (1 / \zeta)-\log ^{\prime}(1 / \zeta-2 \zeta) \cdot 2 \zeta \\
& =\log (1 / \zeta)-2 \zeta \frac{\zeta}{1-2 \zeta^{2}} \geq \log (1 / \zeta)-\frac{2}{1-1 / 8} \zeta^{2} \\
& \geq \log (1 / \zeta)-3 \zeta^{2}
\end{aligned}
$$

so we obtain $\log (1 / \zeta)-3 \zeta^{2} \leq \operatorname{Arcosh}(1 / 2 \zeta) \leq \log (1 / \zeta)$. It follows that

$$
\cos (2 \mu \operatorname{Arcosh}(1 / 2 \zeta))=\cos (2 \mu \log (\zeta))+R_{6}(\mu, \zeta), \quad \text { where }\left|R_{6}(\mu, \zeta)\right| \leq 6 \mu \zeta^{2}
$$


Combining the approximations for the sinh- and the cos-term, we get for $\mu \in(0,2)$ and $\zeta \in(0,1 / 4)$

$$
\begin{aligned}
\psi(\mu, \zeta) & =\left[2 \mu \zeta^{2}+R_{5}(\mu, \zeta)\right] \cdot\left[\cos (2 \mu \log (\zeta))+R_{6}(\mu, \zeta)\right] \\
& =2 \mu \zeta^{2} \cos (2 \mu \log (\zeta))+R_{7}(\mu, \zeta), \quad \text { with } \\
\left|R_{7}(\mu, \zeta)\right| & \leq 2 \mu \zeta^{2} R_{6}(\mu, \zeta)+R_{5}(\mu, \zeta)\left(1+R_{6}(\mu, \zeta)\right) \\
& \leq 2 \mu \zeta^{2} 6 \mu \zeta^{2}+16 \mu \zeta^{4}\left(1+6 \mu \zeta^{2}\right) \\
& \leq 12 \mu^{2} \zeta^{4}+32 \mu \zeta^{4} \leq 56 \mu \zeta^{4} .
\end{aligned}
$$

In particular, we have $|\psi(\mu, \zeta)| \leq 2 \mu \zeta^{2}|\cos (\cdots)|+56 \mu \zeta^{4} \leq 6 \mu \zeta^{2}$.

Finally, noting that $\mu=\mu^{(2 n+1) \tau_{n}}$, we can compute

$$
\begin{aligned}
G_{n}(\mu, \zeta) & =\mu^{2 \tau_{n}}+(-1)^{n+1} 2 \mu^{\tau_{n}}\left[2 \mu \zeta^{2} \cos (2 \mu \log \zeta)+R_{7}(\mu, \zeta)\right]-1 \\
& =\mu^{2 \tau_{n}}+(-1)^{n+1} 4 \mu^{(2 n+2) \tau_{n}}\left[\zeta^{2} \cos (2 \mu \log \zeta)+\frac{R_{7}(\mu, \zeta)}{2 \mu}\right]-1 \\
& =\mu^{2 \tau_{n}}\left\{1+(-1)^{n+1} 4 \mu^{2 n \tau_{n}} \zeta^{2}[\cos (2 \mu \log \zeta)+R(\mu, \zeta)]\right\}-1,
\end{aligned}
$$

where $R(\mu, \zeta):=R_{7}(\mu, \zeta) /\left(2 \mu \zeta^{2}\right)$ and $|R(\mu, \zeta)| \leq 56 \mu \zeta^{4} /\left(2 \mu \zeta^{2}\right)=28 \zeta^{2}$.

b) Claim 1: $\psi$ is bounded on $(0,2) \times(0,1 / 4)$.

This follows from the boundedness of arctan and cos, and from formula (5.2.1) for $\psi$.

Claim 2: $\psi(\mu, \zeta) \rightarrow 0$ and $\partial_{1} \psi(\mu, \zeta), \partial_{2} \psi(\mu, \zeta) \rightarrow 0$ as $\zeta \rightarrow 0, \zeta>0$, uniformly with respect to $\mu \in(0,2)$.

From the above approximation of $\psi$ we know that $|\psi(\mu, \zeta)| \leq 6 \mu \zeta^{2}$ for all $(\mu, \zeta) \in$ $(0,2) \times(0,1 / 4)$, which implies the assertion for $\psi$. Further,

$$
\partial_{1} \psi(\mu, \zeta)=2 \varphi(\zeta) \cosh (2 \mu \varphi(\zeta)) \cos (\cdots)-\sinh (2 \mu \varphi(\zeta)) \sin (\cdots) 2 \operatorname{Arcosh}(1 / 2 \zeta) .
$$

Using the estimates from the proof of b) for $\varphi$, and for the sinh-and the Arcosh-term, we get

$$
\left|\partial_{1} \psi(\mu, \zeta)\right| \leq 2\left(\zeta^{2}+7 \zeta^{4}\right) \cosh (\mu \varphi(\zeta))+\left(2 \mu \zeta^{2}+16 \mu \zeta^{4}\right) 2 \log (1 / \zeta) .
$$

The assertion for $\partial_{1} \psi$ now follows from the boundedness of the cosh-term on $(0,2) \times(0,1 / 4)$. Next, we have

$\partial_{2} \psi(\mu, \zeta)=\cosh (2 \mu \varphi(\zeta)) 2 \mu \varphi^{\prime}(\zeta) \cos (\cdots)-\sinh (2 \mu \varphi(\zeta)) \sin (\cdots) 2 \mu \operatorname{Arcosh}^{\prime}(1 / 2 \zeta) \frac{-1}{2 \zeta^{2}}$.

Note that $w(\zeta)=\sqrt{1-4 \zeta^{2}}$, that $w$ has a maximum at 0 , and hence $\varphi$ has a minimum at $\zeta=0$, so $\varphi^{\prime}(0)=0$. Further,

$$
\operatorname{Arcosh}^{\prime}(1 / 2 \zeta)=\frac{1}{\sqrt{(1 / 2 \zeta)^{2}-1}}=\frac{2 \zeta}{\sqrt{1-4 \zeta^{2}}} .
$$

Writing $T_{1}(\mu, \zeta)$ for the first term and $T_{2}(\mu, \zeta)$ for the second term in the expression for $\partial_{2} \psi(\mu, \zeta)$, it follows from $\varphi^{\prime}(0)=0$ and from the boundedness of the cosh-term that 
$T_{1}(\mu, \zeta) \rightarrow 0$ if $\zeta \rightarrow 0, \zeta>0$, uniformly for $\mu \in(0,2)$. Finally, using the estimate on the sinh-term obtained in the proof of $b$ ) again, we get

$$
\left|T_{2}(\mu, \zeta)\right| \leq\left(2 \mu \zeta^{2}+16 \mu \zeta^{4}\right)|\sin (\cdots)| 2 \mu \frac{2 \zeta}{\sqrt{1-4 \zeta^{2}}} \frac{1}{2 \zeta^{2}} \leq \frac{2 \mu\left(2 \mu \zeta+16 \mu \zeta^{3}\right)}{\sqrt{3 / 4}},
$$

which proves the analogous convergence property for $T_{2}$, and hence for $\partial_{2} \psi$. Claim 2 is proved.

It follows from Claim 2 and from the definitions of $G_{n}$ and $\tilde{G}_{n}$ that $\tilde{G}_{n}$ is continuous and has continuous first partial derivatives, so $\tilde{G}_{n}$ is $C^{1}$.

5.3. Corollary. There exists $\underline{z} \in(1 / 2,1)$, a neighborhood $U$ of 1 in $\boldsymbol{R}$, and a $C^{1}$ function $\lambda^{*}:(\underline{z}, 1) \rightarrow U \subset \boldsymbol{R}$ such that for all $z \in(\underline{z}, 1), \lambda^{*}(z)$ is a semi-Floquet multiplier of $x_{z, n}$, and the only one contained in $U$. Further, $\lambda^{*}(z) \rightarrow 1$ as $z \rightarrow 1$.

Proof. With $\tilde{G}_{n}$ from Lemma 5.2b), note that $\tilde{G}_{n}(1,0)=0$ and $\partial_{1} \tilde{G}_{n}(1,0)=2 \tau_{n} \neq$ 0 . It follows from the Implicit Function Theorem that there exist $\delta>0$, an open neighborhood $\tilde{U} \subset(0,2)$ of 1 in $\boldsymbol{R}$, and a $C^{1}$ function $\mu^{*}:(-\delta, \delta) \rightarrow \tilde{U} \subset \boldsymbol{R}$ such that one has for $\zeta \in(-\delta, \delta)$ and $\mu \in \tilde{U}$ :

$$
\tilde{G}(\mu, \zeta)=0 \Leftrightarrow \mu=\mu^{*}(\zeta) .
$$

Set now $\underline{z}:=1-\delta$, and $U:=\tilde{U}^{-2 \tau_{n}}$, and define $\lambda^{*}(z):=\left(\mu^{*}(1-z)\right)^{-2 \tau_{n}}$ for $z \in(\underline{z}, 1)$. Then, for these $z$, the definitions of $\tilde{G}$ and of $G$ imply

$$
\left(\mu^{*}(1-z)\right)^{2 \tau_{n}} r_{z, n}\left(\lambda^{*}(z)\right)=G_{n}\left(\mu^{*}(1-z), 1-z\right)=\tilde{G}_{n}\left(\mu^{*}(1-z), 1-z\right)=0 .
$$

Hence $\lambda^{*}(z)$ is a semi-Floquet multiplier of $x_{z, n}$. It is the only semi-multiplier in $U$, since for every other multiplier $\lambda \in U$, one would have $\mu:=\lambda^{-(2 n+1) / 2} \in \tilde{U}$ and $\tilde{G}(\mu, 1-z)=$ $G(\mu, 1-z)=r_{z, n}(\lambda) / \mu^{2 \tau_{n}}=0$, so $\mu=\mu^{*}(1-z)$ and $\lambda=\left(\mu^{*}(1-z)\right)^{-2 \tau_{n}}=\lambda^{*}(z)$.

We can now show that, similar to the much more difficult result obtained in [2] for smooth nonlinearities, the value of $\lambda^{*}(z)$ oscillates about 1 , as $z \rightarrow 1$.

5.4. THEOREM. There exists a sequence $\left(z_{k}\right)_{k \in N}$ in $(\underline{z}, 1)$ with $z_{k}<z_{k+1}$ and $z_{k} \rightarrow 1$, and with the following property: The positive semi-Floquet multiplier $\lambda^{*}(z)$ of the rapidly oscillating periodic solution $x_{z, n}$ from Corollary 5.3 satisfies

$$
\lambda^{*}\left(z_{2 k-1}\right)<1<\lambda^{*}\left(z_{2 k}\right) \text { for all } k \in N .
$$

Proof. Recall the function $\mu^{*}$ defined on $(-\delta, \delta)$ from the proof of Corollary 5.3, with $\tilde{G}_{n}\left(\mu^{*}(\zeta), \zeta\right)=0$. In case $\zeta>0$, we have also $G\left(\mu^{*}(\zeta), \zeta\right)=\tilde{G}\left(\mu^{*}(\zeta), \zeta\right)=0$. Since $\mu^{*}(\zeta) \rightarrow 1$ for $\zeta \rightarrow 0$, the function $\zeta \mapsto\left|\mu^{*}(\zeta) \log (\zeta)\right|$ converges to infinity for $\zeta \rightarrow 0, \zeta>0$. It follows that there exists a sequence $\left(\zeta_{k}\right)_{k \in N}$ in $(0, \delta)$ with the properties

$$
\zeta_{k}>\zeta_{k+1}, 28 \zeta_{k}^{2} \leq 1 / 2, \cos \left(2 \mu^{*}\left(\zeta_{k}\right) \log \left(\zeta_{k}\right)\right)=(-1)^{n+1+k} \quad(k \in N) .
$$


It follows from these properties, together with the estimate on $R$ from Lemma 5.2 a), that for $k \in N$ one has

$$
\begin{aligned}
& \operatorname{sign}\left\{(-1)^{n+1} 4\left(\mu^{*}\left(\zeta_{k}\right)\right)^{2 n \tau_{n}} \zeta_{k}^{2}\left[\cos \left(2 \mu^{*}\left(\zeta_{k}\right) \log \zeta_{k}\right)+R\left(\mu^{*}\left(\zeta_{k}\right), \zeta_{k}\right)\right]\right\} \\
& \quad=(-1)^{n+1}(-1)^{n+1+k}=(-1)^{k} .
\end{aligned}
$$

Now $G_{n}\left(\mu^{*}\left(\zeta_{k}\right), \zeta_{k}\right)=0$ and Lemma 5.2 a) imply $\left(\mu^{*}\left(\zeta_{k}\right)\right)^{2 \tau_{n}}=1 /(1+[\ldots])$, where the sign of the bracket alternates with $k$. We see that $\mu^{*}\left(\zeta_{k}\right)>1$ for odd $k$ and $\mu^{*}\left(\zeta_{k}\right)<1$ for even $k$. Setting $z_{k}:=1-\zeta_{k}$, the result now follows from $\bar{z}=1-\delta$ and from $\lambda^{*}\left(z_{k}\right)=\left(\mu^{*}\left(\zeta_{k}\right)\right)^{-2 \tau_{n}}$.

REMARKS AND NUMERICAL OBSERVATIONS. The results of this section can certainly be extended to smooth nonlinearities close to our piecewise linear example. We did not include such technical steps.

Theorem 5.4 above describes the oscillation around 1 of the real positive semi-multiplier $\lambda^{*}(z)$ as $z \rightarrow 1$.

We know from Section 4 that for $z \rightarrow 1$ the semi-multipliers of $x_{z, n}$ converge to the heart-shaped region $\mathcal{H}$. Unfortunately, this fact does not imply that for $z$-values close to 1 and such that $\lambda^{*}(z)<1$, the solutions $x_{z, n}$ are stable: The closure of $\mathcal{H}$ contains -1 , and convergence to $\mathcal{H}$ does not exclude multipliers outside the unit circle.

In fact, for the case $n=1$ we searched for semi-multipliers numerically, employing the explicit expression for the characteristic function and a Newton procedure. We found that for all values of $z$ which we could numerically treat, there exist non-real semi-multipliers outside the unit circle. More specifically, inspecting the range $z \in I:=[0.8,0.9999]$, we found $\lambda^{*}(z)<1$ in the intervals

$$
I_{1}:=[0.8,0.906], I_{2}:=[0.9804,0.9959], I_{3}:=[0.9992,0.9998],
$$

and $\lambda^{*}(z)>1$ in the complementary subintervals of $I$. (z-values larger than 0.9999 were essentially beyond our numerical resolution, and $\lambda^{*}(z)$ converges rapidly to 1 for such $z$.) We found complex semi-multipliers outside the unit circle approximately equal to $0.95 \pm 0.5 i$ for $z \in I_{1}$, to $0.7 \pm 0.75 i$ for $z \in I_{2}$, and to $0.6 \pm 0.8 i$ for $z \in I_{3}$. (These values are only crude approximations, since the precise values change with $z$.) In particular, the solutions $x_{z, 1}$ with $\lambda^{*}(z)<1$ are nevertheless unstable.

It could be that one can change the equation slightly in a way that forces the complex multipliers into the unit circle, and thus obtain stable rapidly oscillating solutions. (This question remains open.) Numerically solving the delay equation from our example, one sees that a slight deviation from the periodic solution typically results in a crossing of the zero 1 (or -1 ) of $f$, and in rapid departure from the values of $x_{z, 1}$.

The numerically observed typical solution behavior of equation $(\alpha f)$ for larger values of $\alpha$ is generally chaotic (compare [8]), and existence of invariant sets with erratic motion was analytically proved in [16] for the specific parameter value $\alpha=(9 / e)(\log 9-1)$ (and small perturbations). 


\section{REFERENCES}

[ 1 ] S.-N. ChOW AND H.-O. WALther, Characteristic multipliers and stability of periodic solutions of $\dot{x}(t)=$ $g(x(t-1))$, Trans. Amer. Math. Soc. 307 (1988), 127-142.

[ 2 ] P. DORMAYER, "Floquet multipliers and secondary bifurcation of periodic solutions of functional differential equations", Habilitation thesis, University of Giessen, 1996.

[ 3 ] P. DORMAYER, Smooth bifurcation of symmetric periodic solutions of functional differential equations, J. Differential Equations 82 (1989), 109-155.

[ 4 ] P. DORMAYER, An attractivity region for characteristic multipliers of special symmetric solutions of $\dot{x}(t)=$ $\alpha f(x(t-1))$ near critical amplitudes, J. Math. Anal. Appl. 169 (1992), 70-91.

[ 5 ] P. DORMAYER, Smooth symmetry-breaking bifurcation for functional differential equations, Differential Integral Equations 5 (1992), 831-854.

[ 6 ] P. DormaYer AND A. F. IVANOv, Stability of symmetric periodic solutions with small amplitude of $\dot{x}(t)=$ $\alpha f(x(t-1))$, Discrete Contin. Dynam. Systems 5 (1999), 61-82.

[ 7 ] P. Dormayer AND A. F. IVANOv, Symmetric periodic solutions of a delay differential equation, Dynamical systems and differential equations, Vol. 1 (Springfield, MO, 1996), Added volume to Discrete Contin. Dynam. Systems, 1998, Added Volume I, 220-230.

[ 8 ] P. DoRmaYer AND B. LANI-WAYDA, Floquet multipliers and Secondary Bifurcations in Functional Differential Equations, Numerical and Analytical Results, Z. Angew. Math. Phys. 46 (1995), 823-858.

[ 9 ] O. Diekmann, S. van Gils, S. Verduyn Lunel and H.-O. Walther, Delay Equations: Complex, Functional, and Nonlinear Analysis, Springer-Verlag, New York, Heidelberg, Berlin, 1995.

[10] A. F. IVANOV AND J. Losson, Stable rapidly oscillating solutions in delay differential equations with negative feedback, Differential Integral Equations 12 (1999), 811-832.

[11] A. F. IVANOV, B. LANI-WAYDA AND H.-O. WALTHER, Unstable hyperbolic periodic solutions of differential delay equations, Recent Trends in Differential Equations (Ed. R. P. Agarwal), 301-316, World Scientific, Singapore, 1992.

[12] A. F. IVANOV AND A. N. Sharkovsky, On periodic solutions of certain classes of differential difference equations, Oscillation and Stability of Solutions of Differential Functional Equations, 25-34, Publ. by the Institute of Mathematics, Ukrainian Academy of Sciences (in Russian), Kiev, 1982.

[13] J. K. Hale And S. Verduyn Lunel, Introduction to Functional Differential Equations, Springer-Verlag, New York, Heidelberg, Berlin, 1993.

[14] G. S. Jones, Periodic motions in Banach space and application to functional differential equations, Contributions to Differential Equations 3 (1964), 75-106.

[15] J. L. KAPLAN AND J. A. YoRKE, Ordinary differential equations which yield periodic solutions of differential delay equations, J. Math. Anal. Appl. 48 (1974), 317-324.

[16] B. LANI-WAYDA, Wandering solutions of sine-like delay equations, Mem. Amer. Math. Soc. 151 No. 718 , Providence, RI, 2001.

[17] R. D. Nussbaum, Uniqueness and nonuniqueness for periodic solutions of $\dot{x}(t)=-g(x(t-1))$, J. Differential Equations 34 (1979), 25-54.

[18] R. D. Nussbaum and H.-O. Peitgen, Special and spurious solutions of $\dot{x}(t)=-\alpha f(x(t-1))$, Mem. Amer. Math. Soc. 51 No. 310, Providence, RI, 1984.

[19] D. SAUPE, Global bifurcation of periodic solutions to some autonomous differential delay equations, Appl. Math. Comput. 13 (1983), 185-211.

[20] A. SChUlZE-HALBERG, Ph-D thesis, in preparation (1999), ETH Zürich

[21] H.-O. WALTHER, Hyperbolic periodic solutions, heteroclinic connections and transversal homoclinic points in autonomous differential delay equations, Mem. Amer. Math. Soc. 79 No. 402, Providence, RI, 1989.

[22] H.-O. Walther, Bifurcation from periodic solutions in functional differential equations, Math. Z. 182 (1983), 269-289. 
MATHEMATISCHES INSTITUT DER JUSTUS-LIEBIG-UNIVERSITÄT ARNDTSTRASSE 2

35392 GIESSEN

GERMANY

E-mail address: Peter.Dormayer@math.uni-giessen.de

MATHEMATISCHES INSTITUT DER JUSTUS-LIEBIG-UNIVERSITÄT

ARNDTSTRASSE 2

35392 GIESSEN

GERMANY

E-mail address: Bernhard.Lani-Wayda@math.uni-giessen.de
DEPARTMENT OF MATHEMATICS

PENNSYlVANIA STATE UNIVERSITY

P.O. BOX PSU

LEHMAN, PA 18627

USA

E-mail address: afi1@psu.edu 